\title{
La réduction des complications de macrosomie, de déchirure périnéale et de césarienne à l'accouchement par la pratique d'activités physiques : état de la recherche
}

\section{Claude E. Hammer M. Lemieux \& G. Kpazaï}

RDRS 2021, 4, pp.87-121

Vivianne Claude, B.EPS (Cand.)

Étudiante au baccalauréat, vclaude@laurentian.ca

École de kinésiologie et des sciences de la santé, Faculté de la santé,

Université Laurentienne, Sudbury (ON), Canada.

Eric Hammer, B.EPS (Cand.)

Étudiant au baccalauréat, ehammer@laurentian.ca

École de kinésiologie et des sciences de la santé, Faculté de la santé,

Université Laurentienne, Sudbury (ON), Canada.

Mikèla Lemieux, B.EPS (Cand.)

Étudiante au baccalauréat, mlemieux1@laurentian.ca

École de kinésiologie et des sciences de la santé, Faculté de la santé,

Université Laurentienne, Sudbury (ON), Canada.

Georges Kpazaï, Ph.D.

Professeur titulaire, gkpazai@laurentienne.ca

École de kinésiologie et des sciences de la santé, Faculté de la santé, Université Laurentienne, Sudbury (ON), Canada.

\section{Résumé}

Au Canada, il y a plus de 350000 accouchements par année (Statistique Canada, 2019). En phase finale de sa grossesse, la femme peut avoir un accouchement avec ou sans complications (OMS, 2018 ; Agence de la Santé publique du Canada, 2018). Selon plusieurs chercheurs, l'activité physique permet, dans plusieurs cas, la prévention de certaines de ces complications (Santé publique Ontario, 2015; Gouvernement du Québec, 2019). La présente étude vise à déterminer, à l'aide d'un état de la recherche, l'apport préventif de l'activité physique en ce qui a trait aux complications de surpoids noé-natal, aux déchirures périnéales ainsi qu'aux césariennes. Par le biais d'analyse de recherche, l'étude cherche à démontrer l'implication de la pratique d'activités physiques dans la réduction des facteurs étiologiques des trois complications. Les résultats obtenus soulignent le rôle clé de prévention d'une intervention basée sur l'activité physique et de loisirs en ce qui concerne le surpoids néo-natal et la césarienne. Quant à l'apport de la pratique d'activités physiques dans la réduction des risques de déchirures périnéales, plus de recherches doivent être menées afin de déterminer si son rôle est significatif.

Mots clés : Complications à l'accouchement, prévention des complications, activités physiques, Macrosomie, déchirure périnéale, césarienne.

\footnotetext{
Abstract

In Canada, there are more than 350,000 childbirths per year (Statistics Canada, 2019). In the final phase of pregnancy, women can have a birth with or without complications (WHO, 2018; Public Health Agency of Canada, 2018). According to several researchers, physical activity in several cases helps prevent some of these complications
} 
Diversity of Research in Health Journal/ Revue de la Diversité de la Recherche en Santé Vol 4, January/ Janvier 2021 - ISSN: 2561 -1666 DOI : 10.28984/drhj.v4i1.335

(Public Health Ontario, 2015; Government du Québec, 2019). The present study aimed to determine whether physical activity plays a part in reducing the etiological factors of three delivery complications: macrosomia, perineal tears as well as caesarean section and, through this analysis, determine whether physical activity acts as a preventative measure. The results obtained underline the key preventative role of a physical activity intervention and of leisure with regard to macrosomia and caesarean section. As for the contribution of physical activity in reducing the risk of perineal tears, more research is needed to determine if its role is significant.

Keywords: Childbirth complications, prevention of complications, physical activity, macrosomia, perineal tear, caesarean section.

\section{Introduction}

Selon plusieurs chercheurs, l'activité physique permet, dans plusieurs cas, la prévention de certaines complications lors de la grossesse. (Santé publique Ontario, 2015 ; Gouvernement du Québec, 2019). Or, la tendance actuelle semble démontrer qu'il y a une augmentation de la sédentarité associée avec la maternité qui passe de 6 à $29 \%$ (Maître, 2013). Avec un changement de perspective, la grossesse peut devenir une opportunité d'apprentissage où, il y a une augmentation de motivation pour adopter des attitudes saines (Kim, Niederdeppe, Guillory, Graham, Olson, et Gay, 2015). Suite à un recensement des écrits, nous avons ressorti quelquesunes des complications les plus prévalentes soit : la césarienne, les accouchements trop longs, la douleur excessive à l'accouchement, la rupture utérine, la déchirure périnéale, la macrosomie, la microsomie ainsi que la mauvaise orientation du bébé. Nous avons choisi de porter notre recherche plus en profondeur sur les trois complications suivantes en fonctions de divers critères.

1.1. Nature et justification du choix de complication

Puisqu'il existe plusieurs complications à l'accouchement et que nous ne pouvions pas toutes les traiter dans cette étude, nous avons fait une sélection discriminante à l'aide de caractéristiques spécifiques telles que : la prévalence, l'absence de recherches approfondies sur la complication et l'ampleur des risques qui y sont associés. Dans la section suivante, nous justifions le choix de porter notre recherche plus en détail sur l'apport de l'activité physique dans la prévention de la césarienne, de la macrosomie ainsi que la déchirure périnéale.

\subsubsection{Césarienne}

Bien qu'elle soit une intervention chirurgicale à l'accouchement, nous la considérons ici comme une «complication». Elle est utilisée pour extraire un bébé par incision du mur de l'abdomen et de l'utérus (Nixon, Berghella, Berens, Cunningham, DeCherney, McInerny, American College of Obstetricians and Gynecologists, 2018). Nous justifions notre choix basé sur les statistiques de prévalence, surtout celles du Canada. Cette optique est double, soit le pourcentage de césarienne actuel ainsi que le quotient d'augmentation des cas. En effet, selon les données empiriques, de plus en plus de femmes ont recours à cette opération pour être capables d'accoucher pour diverses raisons. Selon l'OMS, les cas d'accouchement par césarienne s'élevaient à 27,9\% en 2015 et selon le Canadian Institute for Health Information (CIHI), ce facteur est passé à 28,2 \% en 2016-2017; ce qui démontre que le taux d'accouchement par césarienne est à la hausse. Il serait donc favorable de minimiser cette prévalence croissante, et ce, par le biais de l'activité physique. 
Diversity of Research in Health Journal/ Revue de la Diversité de la Recherche en Santé Vol 4, January/ Janvier 2021 - ISSN: 2561 -1666

DOI : $10.28984 /$ drhj.v4i1.335

\subsubsection{Déchirures périnéales}

Les déchirures périnéales sont des déchirures ou des blessures à la peau et/ou aux muscles entre l'introîtus vaginal et l'ouverture anale. Elles se produisent pendant l'accouchement par voie naturel. En 2011, le taux brut de lésions obstétricales et sphinctériennes au Canada, lors d'accouchements vaginaux pratiqués au moyen d'instruments, était de $17 \%$ (Institut canadien pour la sécurité des patients, 2018). Nous avons sélectionné les déchirures périnéales, comme complication à étudier, pour deux raisons majeures. Premièrement, les déchirures périnéales sont très peu connues et ce sujet tabou peut mettre mal à l'aise une femme souffrant de cette complication. Ce qui peut nuir à son épanouissement social (Skinner et Dietz, 2015). En deuxième lieu, il s'agit d'un problème qui est mentionné dans la littérature, mais qui n'est pas exploré en profondeur. (Borraccetti, 2016). De plus, l'apport que joue l'activité physique semble être encore ambigu ou peu connu. Mentionnons aussi que ce manque de recherches est aussi possiblement influencé par la perception des tabous associés à cette complication. Compte tenu de ce qui précède, il est nécessaire que des recherches plus approfondies soient effectuées spécifiquement au niveau des bienfaits de l'activité physique sur les déchirures périnéales.

\subsubsection{Macrosomie}

La macrosomie occasionne aussi une complication à l'accouchement. Car, elle se produit lorsque le nouveau-né a un poids égal ou supérieur à 4000 g à la naissance (Ridha et col 2017). Selon Statistique Canada, sa prévalence se situait à 1,4\% en 2017. Ses conséquences sont associées autant chez la mère (hémorragie, rupture utérine, allongement du travail) que chez le fœtus (asphyxie, dystocie de l'épaule, fractures) (Ridha, Houssem, Latifa, Ines, et Sabra, 2017 ; Santé publique Ontario, 2015). Par ailleurs, selon les données empiriques, une pratique d'activité physique peut réduire les risques jusqu'à $31 \%$ (Vargas-Terrones, Nagpal et Barakat, 2019); ce qui est énorme. Compte tenu de l'ampleur des risques associés à la macrosomie, il serait crucial de réduire cette complication. D'où l'importance d'avoir plus de recherches sur les bienfaits prophylactiques de l'activité physique maternelle sur la macrosomie.

\subsubsection{Exclusion des autres complications}

En prenant appui sur la littérature scientifique, certaines complications à l'accouchement ont été écartées de notre recherche. Les complications dans lesquelles l'activité physique ne jouait qu'un rôle négligeable ont été mises de côté soit, la microsomie ainsi que la mauvaise orientation du fœtus (Vargas-Terronnes, 2019; Osborne, 2011). Ensuite, les ruptures utérines ont une prévalence moindre avec 0,5\% (Langlois, 2014). C'est pourquoi nous ne nous sommes pas penchés sur cette question.

\subsection{Problématique}

Les trois complications sélectionnées ont des conséquences importantes que ce soit par leur prévalence ou par leur effet sur la santé. De ce fait, il serait bénéfique pour la population de femmes enceintes d'être outillées pour minimiser les risques de souffrance. Une intervention qui semble avoir un rôle préventif selon la littérature est la pratique d'activités physiques lors de la grossesse. Selon les Directives canadiennes en matière d'activité physique lors de la grossesse (2019) les indications sont 150 minutes par semaines. À l'aide d'une recherche documentaire, 
notre objectif est d'identifier des programmes d'activités physiques adaptés à cette population spéciale (femmes enceintes) pour permettre la réduction des risques de complications à l'accouchement qui ont été sélectionnés

\section{Méthodologie}

2.1. Stratégies de collecte des données.

Afin de faire un état de la recherche valide, nous avons utilisé un cadre méthodologique fondé sur une revue de littérature. Le processus se divise en deux étapes. Pour ce qui en est des stratégies de collecte des données, nous avons concentré notre recherche sur des articles traitant de la pratique d'activités physiques chez les femmes enceintes parus entre 2010 et 2020. Les articles ont été trouvés dans les banques de données suivantes PubMed, Google Scholar, Cochrane Library, Science Direct et CINAHL (Cumulative Index to Nursing and Allied Health Literature). Nous avons utilisé les mots clés : Césarienne, activité physique, grossesse, prévention des complications, Cesarean section, physical activity, during pregnancy, adapted physical activity, macrosomie, macrosomia, prévalence, gymnastique périnéale et déchirures périnéales. 436 articles ont été ressortis et 31 ont été retenus. Nous n'avons gardé que les articles qui portaient sur une des trois complications et l'activité physique. Pour cette dernière composante, nous nous sommes intéressés aux articles qui présentaient des résultats sur l'apport de l'activité physique sur la réduction des complications ou les indications en matière d'interventions pour cette population spéciale.

2.2. Stratégies d'analyse des données

L'analyse des données s'est faite en suivant plusieurs étapes. Premièrement, nous avons fait la lecture approfondie de chaque article afin de bien les comprendre et d'en ressortir les informations les plus pertinentes au regard de l'objet de notre recherche. Puis, il y eut la classification des articles lus en fonction de leur pertinence au regard des différents axes de notre question de recherche. Nous avons indiqué le contenu traité par chaque article en fonction des sections consignées dans un tableau synthèse selon les catégories suivantes : nature, étiologie, prévalence, caractéristiques et interventions individualisées. Ceci a facilité la compréhension du handicap, les effets de la pratique d'activités physiques sur les différentes complications à l'accouchement et son rôle dans les stratégies d'interventions spécifiques.

Ensuite, le processus de théorisation nous a permis de faire un état de la recherche concernant les différentes variables mentionnées plus haut. L'étiologie de chaque complication est une information que nous avons analysée en profondeur pour déterminer ce qui pouvait être influencé par la pratique d'activités physiques. Finalement, suite à l'analyse élaborée des articles nous avons ressorti les éléments importants pour définir les stratégies et les paradigmes d'intervention adaptés les plus efficaces.

\section{Résultats}

\subsection{La césarienne}

- Nature de la césarienne

\section{Les complications chez la mère, le foetus et la famille}

Tout comme la macrosomie, les risques sont attribués à la mère et le nouveau-né. Chez la mère, la mortalité et la morbidité sont les risques les plus graves. Il y a aussi des risques à court terme comme l'infection et l'hémorragie sévère ; puis à long terme, une rupture utérine 
(Domenjoz et al., 2014). La césarienne peut aussi augmenter les risques de complications opératoires telles que : la plaie digestive, la plaie de la vessie, une plaie de l'uretère et de déchirure du ligament large (Beucher, Dolley, Lévy-Thissier, Florian et Dreyfus, 2012). Selon la recherche d'Alfirevic, Milan et Livio (2013), il y a 3 à 5 fois plus de risque de mortalité maternelle et 2 fois plus d'hystérectomie dans le cas d'une césarienne. Ils ont aussi mentionné qu'il y a des risques d'infection, de caillots sanguins, d'embolie pulmonaire et une perte sanguine excessive. Cependant, si la mère a déjà eu recours à une césarienne, elle bénéficierait plus d'avoir une deuxième césarienne que d'accoucher sans chirurgie (Beucher et al., 2012). Finalement, dans une autre recherche, Barakat et al. (2012) mettent en évidence que suite à une césarienne, des troubles respiratoires et des problèmes de placenta peuvent subvenir lors des accouchements subséquents. L'étiologie de la césarienne

La césarienne est une procédure utilisée par plusieurs afin d'éviter ou de résoudre diverses complications. Deux causes ressortent de la littérature consultée : l'âge et le surpoids/IMC (Barakat et al., 2012; Neilson, Anderson, Hegaard et Juhl, 2017 ; Morgan, Rahman, Hill, Zhou, Bijlsma, Khanom, Lyons et Brophy, 2014 ; Domenjoz et al., 2014). Barakat et al. (2012) indiquent qu'une femme sédentaire a 2,05\% plus de risque de devoir faire appel à un accouchement par césarienne. Cependant, l'âge avancé et la primiparité (une mère qui a accouché une fois) augmentent les chances de césarienne (Tinloy, Chuang, Zhu, Pauli, Kraschnewski et Kjerulff, 2013 ; Domenjoz, 2014). The American College of Obstetricians and Gynecologists, Society for Maternal-Fetal Medicine présente quelques complications qui peuvent entraîner le recours à une césarienne telles que la difficulté du travail, une mauvaise orientation fœtale et la macrosomie. (Caughey, Cahill, Guise et Rouse, 2014)

\section{L'apport de l'activité physique}

La littérature scientifique relative à la prévention de la césarienne traite abondamment du rôle essentiel des effets de l'activité physique sur cette question. Elle joue aussi un rôle positif dans la réduction d'une intervention par césarienne à l'accouchement (Barakat et al., 2012). Dans son article, Tinloy (2013) met en évidence l'augmentation des risques de césariennes de 5,5 $\%$ chez les femmes qui n'atteignent pas les 150 min d'activités physiques par semaine. Morgan, (2014) a trouvé les mêmes résultats dans sa recherche sur la relation entre l'exercice et la réduction de la césarienne. Néanmoins, il n'existe pas de consensus entre les chercheurs sur ce constat. En effet, Domenjoz (2014) souligne que les risques de césarienne sont minimisés chez la femme si elle pratique de l'activité physique, même si celle-ci n'atteint pas la recommandation de 150 minutes par semaine.

Intervention spécifique

Dans l'étude de Barakat et al. (2012), une intervention en conditionnement physique a été mise en œuvre chez une population (290) de femmes enceintes (120-150 minutes à > $70 \%$ de la fréquence cardiaque maximale). Suite à cette intervention, le taux de césarienne était de 7,1\% plus élevé dans le groupe des femmes sédentaires. De plus, dans le groupe actif avait un taux plus élevé de naissances sans procédure chirurgicale ni instrument (72,5\%). Finalement, Szumilewicz et ses collaborateurs (2013) attirent l'attention sur la contribution de l'activité 
physique non seulement à la santé physique de la mère, mais aussi à sa santé psychologique. Effectivement, suite à leurs interventions, ils ont mis en évidence que les femmes qui font de l'activité physique développent une forte confiance en soi vis-à-vis de leurs capacités d'accoucher par la voie basse. Par conséquent, diminuent le risque d'avoir une intervention par césarienne.

\section{Paradigme d'intervention et stratégies}

Selon l'état de la recherche sur l'intervention éducative auprès d'une population spéciale, le paradigme d'intervention basé sur l'empowerment et la détermination de soi est le plus utilisé (De Blake D. Poland, Lawrence W. Green, Irving, 2000). Ce paradigme d'intervention se traduit auprès des femmes enceintes, selon plusieurs études, par un changement d'attitudes et de croyances face à la pratique de l'activité physique, une fixation d'objectifs, une autosurveillance ainsi qu'une auto-évaluation de celles-ci (Kim, 2015). Comme mentionné plus haut, il y a plusieurs facteurs étiologiques de nature physiologique, psychologique et sociologique (Kim, 2015 ; Santé publique Ontario, 2015 ; Ridha 2017 et Vargas-Terrones, 2019) et ceux-ci doivent être pris en considération dans le processus de programmation. Cela va sans dire que face à la pratique d'activités physiques, plusieurs femmes ont peur pour leur bien-être et celui de leur enfant (Vargas-Terrones, 2019). Or, comme mentionnés plus haut, les risques sont minimes. Une des craintes les plus souvent soulevées par les femmes est que le fœtus allie un poids gestationnel trop petit $(<2500 \mathrm{~g})$. Toutefois, Vargas-Terronnes (2019) n'a trouvé aucune corrélation avec la pratique d'activité physique. Le manque de circulation sanguine et de nutriments qui se rend au fœtus est une des inquiétudes soulevées par les femmes dans l'étude de Szumilewicz (2013). Or, ceci n'est pas supporté par la recherche, même si la circulation placentaire diminue lors de la pratique d'activités physiques. À contrario, le volume sanguin fœtal augmente à long terme. De plus, dans ce même article (Szumilewicz, 2013), il est mis en évidence l'importance d'une intervention qui est bien structurée, mais surtout bien ajustée avec les mesures préventives nécessaires pour répondre aux besoins uniques de la future mère. Compte tenu de l'information fournie, il est crucial de placer l'intervention dans un paradigme humaniste qui est centrée sur les besoins de la participante et qui vise son autonomie (Kpazaï, 2020). C'est dans ce sens que l'étude de Kim (2015) révèle que les deux éléments les plus importants pour le contrôle du poids gestationnel sont: la perception qu'à la future mère par rapport à l'effet que va entraîner l'activité physique sur l'état de santé de son enfant et l'auto-surveillance.

\subsection{Les déchirures périnéales}

- Nature de la macrosomie

Les complications chez la mère, le foetus et la famille

Il a quatre degrés de déchirure, chaque degré est défini selon leur niveau de profondeur. Les effets secondaires des déchirures périnéales se résument souvent à l'incontinence urinaire et/ou fécale. Selon le degré de gravité de la déchirure, la durée peut varier et même être persistante. Dans certains cas, cela affecte la sexualité de la femme. Ce sont tous des problèmes qui peuvent ébranler l'image de soi de celle-ci (Borraccetti, 2016). 


\section{L'étiologie des déchirures périnéales}

Le facteur de risques qui est le plus associés aux déchirures périnéales est un manque d'élasticité des tissues de cette région résultant en grande partie de l'hérédité (Lowdermilk et al., 2012). La position de la mère, dont la forme du bassin est aussi un facteur étiologique (ex : arc sous-pubien étroit avec un détroit inférieur étranglé). La position du fotus est aussi un facteur étiologique, quand celle-ci est anormale (ex : présentation du dos du fotus vers + vertèbre de la mère). En plus, la macrosomie (poids élevé, > 4000g), les forceps et/ou ventouse, la détresse fœtale, un long $2 \mathrm{e}$ stade de travail lors de l'accouchement et le travail rapide sont aussi des facteurs étiologiques (Lowdermilk et al., 2012).

\section{L'apport de l'activité physique}

À l'aide d'une revue de littérature, des chercheurs (Szumilewicz, Wojtyla, Zarebska, DrobnikKozakiewicz, Sawczyn, et Kwitniewska, 2013) ont démontrée, contrairement à ce que soutiennent plusieurs études, que l'activité physique et la pratique d'exercices du plancher pelvien ne causent pas une augmentation de la résistance des muscles périnéales. En effet, dans leur étude, le groupe de femmes qui a participé à l'expérience avait un taux de périnée intact à $36,8 \%$ comparé aux femmes à $23,8 \%$ chez les femmes sédentaires. Or, ces taux ne sont pas assez élevés pour être significatifs. Manifestement, les sessions d'exercice chez les femmes enceintes diminuent la prévalence des déchirures périnéales. Toutefois, cela n'influence pas la longueur du travail lors de l'accouchement. Par ailleurs, certains auteurs affirment que l'entraînement ne fait pas de différence sur les différents degrés de déchirures périnéales et de l'épisiotomie. Par contre, l'activité physique est importante pour garder une bonne santé et une force abdominale. Ce qui facilite la femme à « pousser» lors de l'accouchement en plus d'avoir un effet protecteur sur le périnée (Borraccetti, 2016; Carini, 2017).

\section{Intervention spécifique}

Selon Borraccetti (2016) et Carini (2017), la gymnastique prénatale est un bon moyen pour éviter les complications de façons générales lors de l'accouchement. En revanche, plus le nombre de séances augmente, moins de déchirures du muscle périnéal sont observées à l'accouchement. Pour ce qui est des recommandations précises, la littérature scientifique est muette envers cette complication. Comme le mentionne Fritel (2010), les moyens de préventions des lésions périnéales sont encore inconnus. Donc, il n'a pas de durée ni un nombre de séances à attendre. Par contre, l'activité physique rend le périnée plus tonique, ce qui augmente le risque de déchirures périnéales (Carini, 2017). Cependant, le retour de la femme à l'activité physique doit inclure le travail du plancher pelvien et de la sangle abdominale. Mais, il est important que les exercices effectués ne constituent pas d'abdominaux dans lesquels, il y a une pression de ses muscles vers le bas (Borraccetti, 2016).

\subsection{La macrosomie}

- Nature de la macrosomie

Les complications chez la mère, le foetus et la famille 
Le poids optimal pour un bébé, selon santé Ontario, se situe entre 2,5 et $4 \mathrm{~kg}$ puisque hors de cette étendue les risques de complications additionnels à l'accouchement sont plus élevés (Ridha et col, 2017). Les risques primaires sont materno-fœtal. En effet, lorsque le bébé est atteint de macrosomie, il y a une augmentation de plusieurs risques dont les plus prévalents chez la mère sont la césarienne $(24,6 \%)$, l'hémorragie ( $8 \%$ ) ainsi qu'un allongement de la deuxième phase du travail (76,3\% de plus de 3 heures). Pour ce qui est du bébé, la complication la plus fréquente qu'entraîne la macrosomie est relié à l'épaule qui reste bloquée derrière l'os pelvien (dystocie des épaules) (3,3 \%) (Beta, 2019). Cette recherche a été évaluée robuste par une échelle de qualité. À long terme, selon Vargas-Terrones et al. (2019), l'enfant pourrait souffrir de problèmes cardiovasculaires, d'obésité ou de diabète. Les effets peuvent se prolonger suite à l'accouchement lorsqu'ils sont de l'ordre des traumatismes génitaux tels que les déchirures périnéales. Il y a même un faible risque d'asphyxie fœetale, susceptible d'entraîner la mort du fœtus (Ridha, 2017 ; Vargas-Terrones, 2019). La famille, avec sa fonction de proche aidant, est aussi grandement affectée par les complications qui affectent la mère et le bébé. En bref, il est évident que les effets secondaires de cette complication ne sont pas bénins.

L'étiologie de la macrosomie

Basés sur la recension des récentes études, plusieurs facteurs sont à l'origine de cette complication. Vargas-Terrones (2019), souligne une multitude de facteurs de risques potentiels, entre autres, un âge maternel élevé, une obésité maternelle (45\%), un terme prolongé (26,9\%) et une multiparité (47\%). Certaines études mentionnent aussi le diabète dans $5 \%$ des cas. Ridha et al. (2017) stipulent que l'indice de masse corporelle (IMC) prégestationnelle (obésité) est un facteur de risque encore plus important que le diabète gestationnel. En outre, dans son étude l'auteur Vargas-Terrones (2019) révèle l'effet du gain de poids gestationnel excessif comme une cause à la macrosomie.

L'apport de l'activité physique

Nous portons notre attention plus spécifiquement sur les facteurs qui peuvent être modifiés par l'exercice. En effet, plusieurs recherches (Vargas-Terrones, 2019; Hoffmann, Günther, Geyer, Stecher, Kunath, Meyer, Spies, Rosenfeld, Kick, Rauh, et Hauner, 2019) identifient l'activité physique maternelle lors de la grossesse comme étant un des facteurs modifiables qui influence positivement le poids à la naissance.

Dans leur étude, les chercheurs Vargas-Terrones et al. (2019) ont démontré un effet positif de l'activité physique lors de la grossesse sur le poids gestationnel par le gain de poids approprié chez la mère. Ceci serait causé par la diminution du stockage de matière grasse chez la mère et du fotus. La pratique d'activités physiques lors de la grossesse diminuerait, selon plusieurs méta-analyses, les risques de macrosomie de 30-31 \% (Mudd, Owe, Mottola et Pivarnik, 2013 ; Vargas-Terrones, 2019). En moyenne, la pratique d'activités physiques à un intervalle aussi minime que 15 minutes 3 fois par semaine (50-60\% de la fréquence cardiaque maximale) va diminuer la masse fœtale de 160 g (Ghodsi et Asltoghiri, 2014). Il convient de souligner que ces études comportent toutefois certaines limites, notamment l'échantillonnage uniquement sud-européen ainsi que l'influence de la désirabilité sociale des réponses en ligne. 
Diversity of Research in Health Journal/ Revue de la Diversité de la Recherche en Santé Vol 4, January/ Janvier 2021 - ISSN: 2561 -1666

DOI : $10.28984 /$ drhj.v4i1.335

Intervention spécifique

Dans plusieurs des études analysées, il est proposé des types spécifiques d'activités physiques qui incluent les indications et contre-indications. Une des interventions de Vargas-Terrones et col. (2019) présente un programme 150 minutes d'exercices (aérobie, renforcement musculaire et flexibilité) par semaine. Une diminution de 2,9\% de macrosomie a été aperçue dans le groupe expérimental par rapport au groupe contrôle.

Plusieurs études proposent la marche, la natation, le vélo stationnaire et autres activités aérobies sans impactes combinées avec de la résistance légère et de la flexibilité, plusieurs fois par semaine (Ghodsi, 2014 ; Maître, 2013 ; Vargas-Terrones et al. 2019). Selon ce qui a été mis en évidence précédemment, le gain de poids gestationnel est un facteur étiologique majeur dans la macrosomie. Dans leur article, Maître et al (2013) recommandent une augmentation de la fréquence cardiaque d'approximativement 60-70\%. Selon une autre recherche, l'effet est plus significatif pour la prévention de la macrosomie par le biais de la perte de poids chez la femme enceinte lorsque l'activité physique est modérée plutôt que léger (Tomic, 2013). Les femmes sont encouragées à prendre conscience des changements qui se produisent dans leurs corps afin de relever l'apparition de certains symptômes contre-indiqués tels que le vertige, des contractions utérines ou la dyspnée (Maître, 2013). En somme, il est évident que les bienfaits de l'activité physique adaptée de façon sécuritaire pour cette population soient indéniables et qu'avec une intervention adéquate les risques materno-fœtal soient minimisés.

\section{Discussion}

Comme observé, la littérature scientifique analysée atteste que l'activité physique semble être une intervention préventive en ce qui a trait aux complications liées à l'accouchement par la césarienne, aux déchirures périnéales ainsi qu'à la macrosomie. Le type d'activités physiques spécifiques recommandée diffère selon la complication, mais la plupart des auteurs reconnaissent son rôle crucial dans la diminution des risques de ces complications à l'accouchement. L'activité physique est centrale dans l'acquisition d'une bonne santé physique et psychologique chez les femmes enceintes. L'activité physique améliore la santé de la mère, car elle diminue inévitablement les risques de complications à l'accouchement (Domenjoz et al., 2014).

Les facteurs de risques prédominants de la macrosomie qui peuvent être modifiés par l'exercice sont tous en corrélation avec le poids corporel, soit l'IMC prégestationnel ou lors de la grossesse (Ridha et col, 2017) ainsi que le gain de poids gestationnel (Kim, 2015 ; Maître, 2013). Ceci étant dit; il serait favorable pour diminuer le risque de la complication de macrosomie, d'avoir comme objectif, lors de la pratique d'activités physiques, une gestion du poids corporel, et ce, à tous les stades de la grossesse. Ceci est mis en évidence par la plupart des études recensées, qui sont fondées sur une démarche de recherche rigoureuse menée auprès de plusieurs participants ainsi que des métas analyses (Kim, 2015 ; Ridha, 2017 ; Mudd, 2013, Beta, 2019). En ce qui concerne les déchirures périnéales, il n'y a pas, à ce jour, assez de recherches approfondies pour appuyer un apport significatif de l'activité physique dans la réduction de cette complication. Il serait donc nécessaire de mener plus d'études sur cette question. Toutefois, 
comme le mentionne (Carini, 2017), l'importance de l'activité physique est démontrée dans le processus de rétablissement suite à une déchirure périnéale. Nous pouvons constater que la pratique quotidienne d'activités physiques, lors de la grossesse, serait favorable pour la femme enceinte puisqu'elle serait ainsi familiarisée avec cette pratique. De plus, ce serait plus facile pour cette femme de poursuivre un programme d'activité physique postpartum visant à la réhabilitation suite à une déchirure périnéale (Kunath, Rauh, Rosenfeld, Kick et Hauner, 2014). Enfin, la contribution de l'activité physique dans la prévention de la césarienne est très bien démontrée (Tinloy, 2013; Morgan, 2014). La littérature a aussi mis en évidence que l'activité physique de haute intensité réduit encore plus le risque de césarienne que l'exercice à basse intensité (Morgan, 2014 ; Neilsen, 2017). De surcroît, Domenjoz (2014) précise la modalité de l'activité physique : l'entraînement de résistance et aérobie réduit le risque par $15 \%$ de césarienne, et en plus, réduit le gain poids gestationnel de $1 \mathrm{~kg}$ en moyenne. La répétition des résultats similaires lors des lectures et analyse des articles met en évidence que les chances de césarienne sont réduites par la pratique d'activités physiques. En fonction des facteurs de risques et le rôle de l'activité physique, les objectifs d'intervention se divisent en deux axes. Premièrement, la gestion d'un poids santé comme dans le cas de la macrosomie (Domenjoz, 2014 ; Hegaard, 2017 ; Morgan, 2014). En second lieu, un apport unique de l'activité physique pour la réduction de cette complication est le développement de la force psychologique et confiance en soi de la femme via ses capacités physiques et la gestion de la douleur. En effet les femmes qui pratiquent de l'activité physique développent leur force physique et émotionnelle. Elles se sentent ainsi prêtes à faire face à l'accouchement (Szumilewicz, 2013).

Nous remarquons que la pratique d'activités physiques diminue le risque de complications spécifiques d'autant plus des risques des variables étiologiques qui sous-tendent ces complications. Il existe une association entre les variables étiologiques et donc une diminution des risques de complications dans son ensemble. À la lumière de l'état de la recherche sur la question, il est évident que les trois complications ne sont pas complètement indépendantes. Pour soutenir ce propos, les études démontrent que la macrosomie est un facteur éthologique autant pour la césarienne que pour les déchirures périnéales (Caughey, 2014 ; Lowdermilk, 2012). C'est pourquoi il nous est possible d'affirmer, à l'instar de Maître (2013), que la pratique d'activités physiques ayant comme finalité la réduction des risques d'une complication spécifique va inévitablement diminuer les risques des autres complications à l'accouchement. Toutefois, il est à noter que d'autres facteurs pourraient biaiser ces résultats notamment la gestion du travail et l'épuisement lors de cette phase, l'âge, l'IMC et la gestation multiple (Caughey et col., 2014). Ridha et col. (2017) affirment que la césarienne serait plus fréquemment utilisée pour des raisons de défauts d'accommodations du fœetus ou difficulté en ce qui a trait aux contractions.

En bref, la motivation et les bienfaits de la pratique de l'activité physique s'étendent à beaucoup plus qu'uniquement la réduction des risques de césarienne, macrosomie et déchirures périnéales (CSEP, 2019; Maître, 2013). Peu importe l'objectif, lorsqu'il est question de programme d'activités physiques pour cette population spéciale, l'important est de débuter par un changement au niveau affectif vis-à-vis de l'apport de l'activité physique dans le processus de 
grossesse, d'accouchement, de maintien de la santé de la future mère et de celle de l'enfant. Peu importe la complication qui est visée, il est important pour les femmes enceintes de devenir autonomes puisque toutes les femmes ont une expérience de grossesse unique (Kim, 2015). L'approche anthropocentrique permettra à la femme enceinte d'être un agent actif dans sa pratique d'activités physiques et le centre de l'intervention. (Amezcua-Prieta, Olmedo-Requena, Jiménez-Mejías, Mozas-Moreno, Lardelli-Claret et Jiménez-Moleon, 2013).

\section{Conclusion}

En ce qui a trait à notre question de recherche portant sur un état de la recherche sur l'apport de l'activité physique sur la réduction des complications à l'accouchement de la macrosomie, de la césarienne et des déchirures périnéales, nos recherches ont démontré le rôle majeur dans les deux premières complications et le besoin de plus d'exploration scientifique pour la dernière complication. Pour les déchirures périnéales, des recherches plus spécifiques sont nécessaires afin d'avoir un aperçu plus approfondi de l'apport de l'activité physique sur cette complication. Les limites de notre étude concernent principalement la grande variété de facteurs étiologiques, les relations entre elles qui ne sont pas toujours bien définies, ainsi que l'interaction bidirectionnelle entre les trois types/catégories de complications. Nous pouvons aussi noter que notre état de la recherche se fonde uniquement sur 31 d'articles scientifiques et donc notre collecte de données est, d'un point de vue quantitatif, relativement faible. De plus, les données des études démontrant l'apport significatif de l'activité physique manquent de précision quant aux différentes intensités, périodes gestationnelles, types d'exercices et différences individuelles, ce qui nous empêche de tirer une conclusion spécifique. Or, même s'il n'y a pas une relation de cause à effet entre l'activité physique et l'éradication des complications à l'accouchement, la littérature scientifique consultée met en évidence la diminution des risques de complications à l'accouchement par la pratique d'activités physiques (Szumilewicz, 2013). Les femmes enceintes qui présentent des facteurs étiologiques de la césarienne et de la macrosomie devraient donc pratiquer l'activité physique pour réduire ces risques. Puisque les bienfaits de l'activité physique sont multiples, les femmes qui n'ont pas de signes de risques de complications devraient, elles aussi, s'adonner à la pratique d'activité physique afin non seulement d'augmenter leur chance d'avoir un accouchement sans accrochage, mais permettre aussi d'améliorer leur santé et celle du fœtus (Kim, 2015). Toutefois, malgré l'existence d'une littérature sur cette question, il reste encore de la place à d'autres recherches afin de suggérer des interventions plus spécifiques en ce qui concerne les types de modalités d'activités physiques pour la réduction des complications à l'accouchement. 
Diversity of Research in Health Journal/ Revue de la Diversité de la Recherche en Santé Vol 4, January/ Janvier 2021 - ISSN: 2561 -1666 DOI : 10.28984/drhj.v4i1.335

\section{Références}

Agence de la santé publique du Canada. (2018). Care during labour and birth. Dans FamilyCentred Maternity and Newborn Care: National Guidelines (p. 81). Repéré à https://www.canada.ca/content/dam/phac-aspc/documents/services/publications/healthyliving/maternity-newborn-care/maternity-newborn-care-guidelines-chapter-4-eng.pdf

Alfirevic, Z., Milan, S. J., \& Livio, S. (2012). Caesarean section versus vaginal delivery for preterm birth in singletons. Cochrane Database of Systematic Reviews, (6), 1-91.

Amezcua-Prieta, C., Olmedo-Requena, R., Jiménez-Mejías, E., Mozas-Moreno, J., LardelliClaret, P., Jiménez-Moleon J.,. (2013). Factors associated with changes in leisure time physical activity during early pregnancy, 121(2), 127131. https://doi.org/10.1016/j.ijgo.2012.11.021

Barakat, R., Pelaez, M., Lopez, C., Montejo, R., \& Coteron, J. (2012). Exercise during pregnancy reduces the rate of cesarean and instrumental deliveries: results of a randomized controlled trial. The Journal of Maternal-Fetal \& Neonatal Medicine, 25(11), 2372-2376.

Beta, J., Khan, N., Khalil, A., Fiolna, M., Ramadan, G., \& Akolekar, R. (2019). Maternal and neonatal complications of fetal macrosomia: Systematic review and meta-analysis. Ultrasound in Obstetrics \& Gynecology, 54(3), 308318. https://doi.org/10.1002/uog.20279

Beucher, G., Dolley, P., Lévy-Thissier, S., Florian, A., \& Dreyfus, M. (2012). Bénéfices et risques maternels de la tentative de voie basse comparée à la césarienne programmée en cas d'antécédent de césarienne. Journal de gynécologie obstétrique et biologie de la reproduction, $41(8), 708-726$.

Borraccetti, V. (2016). La rééducation périnéale. Repéré à http ://www.chu-tivoli.be/wpcontent/uploads/2015/12/la-re\%CC\%81 \%CC\%81ducationpe\%CC\%81rine\%CC\%81ale_-Vera-Borraccetti.pdf

Canadian Institute for Health Information (2019) C-section rates continue to increase while birth rates decline. Retrouver le $1^{\text {er }}$ mars 2020 à https ://www.cihi.ca/en/c-section-ratescontinue-to-increase-while-birth-rates-decline

Carini, A. L. (2017). Activité physique chez la femme enceinte : représentations et pratiques professionnelles des sages-femmes de Nice. https ://pdfs.semanticscholar.org/a437/53af3be8790d3d46c573771409887b7633b4.pdf

Caughey, A. B., Cahill, A. G., Guise, J. M., Rouse, D. J., \& American College of Obstetricians and Gynecologists. (2014). Safe prevention of the primary cesarean delivery. American journal of obstetrics and gynecology, 210(3), 179-193.

CSEP (Société canadienne de physiologie de l'exercice). (2019.). Édition 2019 des Directives canadiennes en matière d'activité physique pendant la grossesse. Repéré à https ://csepguidelines.ca/wp-

content/uploads/2018/10/4208_CSEP_Pregnancy_Guidelines_Fr_P2A.pdf 
Diversity of Research in Health Journal/ Revue de la Diversité de la Recherche en Santé Vol 4, January/ Janvier 2021 - ISSN: 2561 -1666 DOI : 10.28984/drhj.v4i1.335

Blake D. Poland, Lawrence W. Green, Irving. (2000). Settings for Health Promotion : Linking Theory and Practice. London : Sage publication inc.

Domenjoz, I., Kayser, B., \& Boulvain, M. (2014). Effect of physical activity during pregnancy on mode of delivery. American journal of obstetrics and gynecology, 211(4), 401-e1.

Ducarme, G., Pizzoferrato, A. C., de Tayrac, R., Schantz, C., Thubert, T., Le Ray, C., et Artzner, F. (2018). Prévention et protection périnéale en obstétrique : Recommandations pour la Pratique Clinique du CNGOF (texte court). Gynécologie Obstétrique Fertilité \& Sénologie, 46(12), 893-899.

Dunkin, M.J., Biddle, B.J. (1974). The study of teaching. New York; Holt Rinehart and Winston. Elizabeth Sinner et Hans Dietz. (2015). Psychological and somatic sequalae of traumatic vaginal delivery: A literature review. Australian and New Zealand Journal of Obstetrics and Gynaecology, 55, 309314. https://doi.org/10.1111/ajo.12286

Fritel, X. (2010). Périnée et grossesse. Gynécologie obstétrique \& fertilité, 38(5), 332-346. GHO (2018) | By category | Births by caesarean section - Data by country. Retrouver le $1^{\text {er }}$ mars 2020 à http ://apps.who.int/gho/data/node.main.BIRTHSBYCAESAREAN?lang=en

Ghodsi, Z., \& Asltoghiri, M. (2014). Effects of aerobic exercise training on maternal and neonatal outcome : A randomized controlled trial on pregnant women in Iran. J Pak Med Assoc, 64(9), 1053-1056

Gouvernement du Québec. (2019). L'activité physique pendant et après la grossesse. Repéré à http ://www.education.gouv.qc.ca/fileadmin/site_web/documents/loisirsport/BrochureActivePourLaVie2019_WEB.PDF

Hélène Langlois. (2014, janvier). L'accouchement vaginal après césarienne (AVAC). Le portail d'information prénatale. Repéré à https ://www.inspq.qc.ca/Data/Sites/8/SharedFiles/PDF/accouchement-vaginal-aprescesarienne-avac.pdf

Hoffmann, J., Günther, J., Geyer, K., Stecher, L., Kunath, J., Meyer, D., Spies, M., Rosenfeld, E., Kick, L., Rauh, K., \& Hauner, H. (2019). Associations between Prenatal Physical Activity and Neonatal and Obstetric Outcomes-A Secondary Analysis of the ClusterRandomized GeliS Trial. Journal of clinical medicine, 8(10), 1735.

Institut canadien pour la sécurité des patients. (2018, octobre). Traumatisme obstétrical : Ressources d'amélioration pour les préjudices à l'hôpital. Repéré à https ://www.patientsafetyinstitute.ca/fr/toolsresources/Hospital-Harm-

Measure/Documents/Resource-Library/HHIR\%20Obstetric\%20Trauma\%20FR.pdf

Kim, H. K., Niederdeppe, J., Guillory, J., Graham, M., Olson, C., \& Gay, G. (2015).

Determinants of Pregnant Women's Online Self-Regulatory Activities for Appropriate Weight Gain. Health Communication, 30(9), 922932.

https ://doi.org/10.1080/10410236.2014.905900

Kunath, J., Rauh, K., Rosenfeld E., Kick L. et Hauner, H. (2014). GeliS: An evaluation of a lifestyle intervention programme on exercise and nutritional habits in pregnant women, families, and infants, 29, S44. https://doi.org/10.1016/j.scispo.2014.08.088 
Diversity of Research in Health Journal/ Revue de la Diversité de la Recherche en Santé

Vol 4, January/ Janvier 2021 - ISSN: 2561 -1666 DOI : 10.28984/drhj.v4i1.335

Lowdermilk, D. L., Perry, S. E., \& Cashion, K. (2012). Soins infirmiers périnatalité. (Édition française: Françoise Courville, Francine de Montigny, Isabelle Milette, Viola Polomeno). Montréal, QC : Chenelière Éducation.

Magro-Malosso, E., Saccone, G., Chen, M., Navathe, R., Di Tommaso, M., \& Berghella, V. (2017). Induction of labour for suspected macrosomia at term in non-diabetic women : A systematic review and meta-analysis of randomized controlled trials. BJOG: An International Journal of Obstetrics \& Gynaecology, 124(3), 414421. https://doi.org/10.1111/1471-0528.14435

Maître, C. (2013). Sport et grossesse : Une nécessaire prescription. Science \& Sports, 28(2), 103108. https ://doi.org/10.1016/j.scispo.2013.01.006

Merriam-Webster. (n.d.). Cesarean section. In Merriam-Webster.com dictionary. Retrouvé le $1^{\mathrm{er}}$ mars 2020 à https ://www.merriam-webster.com/dictionary/cesarean\%20section

Morgan, K. L., Rahman, M. A., Hill, R. A., Zhou, S. M., Bijlsma, G., Khanom, A., Lyons, R. A.,. \& Brophy, S. T. (2014). Physical activity and excess weight in pregnancy have independent and unique effects on delivery and perinatal outcomes. PloS One, 9(4). https ://www.ncbi.nlm.nih.gov/pubmed/24722411

Mudd, L. M., Owe, K. M., Mottola, M. F., \& Pivarnik, J. M. (2013). Health Benefits of Physical Activity during Pregnancy: An International Perspective. Medicine \& Science in Sports \& Exercise, 45(2), 268277. https ://doi.org/10.1249/MSS.0b013e31826cebcb

Nielsen, E. N., Andersen, P. K., Hegaard, H. K., \& Juhl, M. (2017). Mode of delivery according to leisure time physical activity before and during pregnancy: a multicenter cohort study of low-risk women. Journal of pregnancy, 2017. https ://www.hindawi.com/journals/jp/2017/6209605

Nixon, H, Berghella, V., Your guide to breastfeeding, Berens, P., Cunningham, FG., DeCherney, AH., McInerny, TK., American College of Obstetricians and Gynecologists. (2018). "CSection." Mayo Clinic, Mayo Foundation for Medical Education and Research, Retrouver le 1er mars 2020 à www.mayoclinic.org/tests-procedures/c-section/about/pac-20393655.

Organisation mondiale de la Santé. (2018). Pour que l'accouchement soit une expérience positive, il est essentiel d'apporter des soins individualisés. Genève. Repéré le 5 avril 2020 à https ://www.who.int/mediacentre/news/releases/2018/positive-childbirthexperience/fr/

Osborne, C., Ecker, J. L., Gauvreau, K., Davidson, K. M., \& Lieberman, E. (2011). Maternal Temperature Elevation and Occiput Posterior Position at Birth Among Low-Risk Women Receiving Epidural Analgesia. Journal of Midwifery \& Women's Health, 56(5), 446451. https://doi-org.librweb.laurentian.ca/10.1111/j.1542-2011.2010.00064.X

Oxford University Press (2010). Caesarean section. In Concise Medical Dictionary: Oxford University Press. Retrouvé le 2 mars. 2020 à https ://www.oxfordreference.com/view/10.1093/acref/9780199557141.001.0001/acref9780199557141-e-1383. 
Diversity of Research in Health Journal/ Revue de la Diversité de la Recherche en Santé Vol 4, January/ Janvier 2021 - ISSN: 2561 -1666 DOI : 10.28984/drhj.v4i1.335

Ridha, F., houssem, R., Latifa, M., Ines, M., \& Sabra, H. (2017). Facteurs de risque et pronostic materno-fœtal de la macrosomie fœtale: Étude comparative a propos de 820 cas. Pan African Medical Journal, 28. https://doi.org/10.11604/pamj.2017.28.126.8508

Santé Publique Ontario. (2015). Evidence Brief. Risk Factors for Large for Gestational Age (LGA) Infants in Ontario.

Shannon E. Perry, Deitra L. Lowdermilk, Kitty Cashion,. (2012). Soins infirmiers-Périnatalité (5e éd.). France : CHENELIÈRE ÉDUCATION.

Simard, C., Caron, F., et Skrosky, K. (Dir.) (1987). Activité physique adaptée. Montréal : Gaetan Morin Éditeur

Skinner, E. M., \& Dietz, H. P. (2015). Psychological and somatic sequelae of traumatic vaginal delivery: A literature review. Australian and New Zealand Journal of Obstetrics and Gynaecology, 55(4), 309314. https://doi.org/10.1111/ajo.12286

Statistique Canada. (2018). Live births, by birth weight. Repéré à https://www150.statcan.gc.ca/t1/tbl1/en/tv.action?pid=1310042201

Statistique Canada. (2019). Estimation des naissances, par sexe, annuelles Repéré à https://www150.statcan.gc.ca/t1/tbl1/fr/tv.action?pid=1710001601

Szumilewicz, A., Wojtyla, A., Zarebska, A., Drobnik-Kozakiewicz, I., Sawczyn, M., et Kwitniewska, A. (2013). Influence of prenatal physical activity on the course of labour and delivery according to the new Polish standard for perinatal care. Annals of Agricultural and Environmental Medicine, 20(2), 380-389 https://www.ncbi.nlm.nih.gov/pubmed/23772595

Tinloy, J., Chuang, C. H., Zhu, J., Pauli, J., Kraschnewski, J. L., \& Kjerulff, K. H. (2014). Exercise during pregnancy and risk of late preterm birth, cesarean delivery, and hospitalizations. Women's Health Issues, 24(1), e99-e104.

Tomić, V., Sporiš, G., Tomić, J., Milanović, Z., Zigmundovac-Klaić, D., \& Pantelić, S. (2013). The effect of maternal exercise during pregnancy on abnormal fetal growth. Croatian Medical Journal, 54(4), 362368 https://doi.org/10.3325/cmj.2013.54.362 
Diversity of Research in Health Journal/ Revue de la Diversité de la Recherche en Santé

Vol 4, January/ Janvier 2021 - ISSN: 2561 -1666 DOI : 10.28984/drhj.v4i1.335

\section{Annexes}

Tableau 1 : Macrosomie

\begin{tabular}{|c|c|c|c|c|c|}
\hline Titre de l'Article & Nature & Étiologie & Prévalence & Caractéristiques (Effets) & intervention \\
\hline 1.Statistiques Canada & $>4000 \mathrm{~g}$ & & $1,4 \%$ & & \\
\hline $\begin{array}{l}\text { 2.Impact of exercise } \\
\text { during pregnancy on } \\
\text { gestational weight gain } \\
\text { and birth weight: an } \\
\text { overview. } \\
\text { (Marina Vargas-Terronesa } \\
\text { et al., 2019) }\end{array}$ & $\begin{array}{l}>4000 \mathrm{~g} \\
>4500 \mathrm{~g} \text { (risques } \\
\text { plus élevés) }\end{array}$ & $\begin{array}{l}\text { - IMC de la mère avant } \\
\text {-Gain de poids maternel } \\
\text { lors de la gestation }\end{array}$ & & $\begin{array}{l}\text { - Augmentation du risque de } \\
\text { césarienne } \\
\text { - Augmentation du risque de ruptures } \\
\text { (utérines) } \\
\text { - Saignements } \\
\text { - Dystocie de l'épaule } \\
\text { - Mort du foetus }\end{array}$ & $\begin{array}{l}\text { - AP permet d'avoir un gain de poids maternel } \\
\text { santé } \\
\text { - AP permet d'avoir un bébé de poids normal } \\
\text {-3 sessions AP (aérobie, flexibilité et } \\
\text { renforcement musculaire) : } 2,3 \% \text { moins } \\
\text { d'incidences de macrosomie } \\
\text {-31\% de diminution de macrosomie avec } \\
\text { l'exercice prénatal } \\
\text { - diminution de la leptine et donc de } \\
\text { l'adiposité fotale } \\
\text { - diminuer les risques de diabète gestationnel } \\
\text { en abaissant le niveau de glucose en } \\
\text { circulation } \\
\text { - Recommandation : AP sécuritaire (marche, } \\
\text { natation, vélo stationnaire, aérobie sans } \\
\text { impact) 20-30min d'activités modérées } \\
\text { plusieurs jours par semaine } \\
\text { - Modifications : mouvements rapides et } \\
\text { positions qui empêchent le retour veineux } \\
\text { (telles que de longues périodes sur le dos) }\end{array}$ \\
\hline $\begin{array}{l}\text { 3.Facteurs de risque et } \\
\text { pronostic mâtereaux } \\
\text { fotaux de la macrosomie } \\
\text { fotale : étude comparative } \\
\text { à propos de } 820 \text { cas } \\
\text { (Fatnassi Ridha et al., } \\
\text { 2017) }\end{array}$ & & $\begin{array}{l}\text { - obésité maternelle } \\
\text { - terme prolongé } \\
\text { - Multiparité } \\
\text { - diabète } \\
\text { - Âge maternel (> } 35 \text { ans) }\end{array}$ & & $\begin{array}{l}\text { - Risques maternel et foetal } \\
\text { - l'augmentation du taux de césarienne } \\
\text { —, d'hémorragie de la délivrance } \\
\text { - déchirures traumatiques cervico- } \\
\text { vaginales } \\
\text { - Épuisement maternel. } \\
\text { - Dystocie des épaules } \\
\text { - augmentation des risques } \\
\text { d'allongement de la deuxième phase du } \\
\text { travail ( }>3 \mathrm{~h} \text { ) de } 29,6 \%\end{array}$ & \\
\hline
\end{tabular}


Diversity of Research in Health Journal/ Revue de la Diversité de la Recherche en Santé Vol 4, January/ Janvier 2021 - ISSN: 2561 -1666 DOI : 10.28984/drhj.v4i1.335

\begin{tabular}{|c|c|c|c|c|}
\hline $\begin{array}{l}\text { 4.Maternal and neonatal } \\
\text { complications of fetal } \\
\text { macrosomia: } \\
\text { systematic review and } \\
\text { meta-analysis } \\
\text { (Beta et al., 2019) }\end{array}$ & $>4000 \mathrm{~g}$ & & $\begin{array}{l}\text { Augmentation des risques maternels } \\
(>4000 \mathrm{~g}) \\
\text { 1.Césarienne d'urgence }(1,98 \%) \\
\text { 2. Hémmoragie postpartum }(2,05 \%) \\
\text { 3.blessure au sphincter anal }(1,91 \%) \\
\text { Fœtal } \\
\text { 4.dystocie de l'épaule }(9,54 \%) \\
\text { 5.blessure au plexus brachial }(11,03 \%) \\
\text { 6. fractures }(6,54 \%) \\
\\
(>4500 \mathrm{~g}) \\
1.2,55 \% \\
2.3,15 \% \\
3.2,56 \% \\
4.15,64 \% \\
5.19,87 \% \\
6.8 .16 \%\end{array}$ & \\
\hline $\begin{array}{l}\text { 5.Induction of labour for } \\
\text { suspected macrosomia at } \\
\text { term in non-diabetic } \\
\text { women: a systematic } \\
\text { review and meta-analysis } \\
\text { of randomized } \\
\text { controlled trials } \\
\text { ( Margo-Malosso, 2016) }\end{array}$ & $>4000 \mathrm{~g} \mathrm{du}$ fœtus & $\begin{array}{l}1-10 \% \text { des } \\
\text { grossesses }\end{array}$ & $\begin{array}{l}\text { - morbidité et mortalité fotal } \\
\text { - accouchement plus long } \\
\text { - dystocie } \\
\text { - operative vaginal delivery } \\
\text { - césarienne } \\
\text { - hémorragies postpartum } \\
\text { - dystocie de l'épaule avec paralysie } \\
\text { bacterial } \\
\text { - asphyxie } \\
\text { Paralysie du nerf faciale }\end{array}$ & \\
\hline 6. Health Benefits of & & & & Limite $140 \mathrm{bpm}$ maternel \\
\hline
\end{tabular}


Diversity of Research in Health Journal/ Revue de la Diversité de la Recherche en Santé Vol 4, January/ Janvier 2021 - ISSN: 2561 -1666 DOI : 10.28984/drhj.v4i1.335

\begin{tabular}{|c|c|c|c|c|c|}
\hline $\begin{array}{l}\text { Physical Activity during } \\
\text { Pregnancy: An } \\
\text { International Perspective } \\
\text { (Mudd et al., 2013) }\end{array}$ & & & & & $\begin{array}{l}\text { AP sur la distribution du sang dans le cordon } \\
\text { ombilical : pas d'effet négatif } \\
\rightarrow \text { diminue durant, mais augmente après } \\
\rightarrow \text { les risques de fausse couche ne sont pas } \\
\text { significatifs } \\
\text { - intensité basse à modérer } 3 / \text { semaines et } \\
\text { d'ajuster en fonction des symptomes } \\
\text { - minimum de } 30 \text { min d'aérobie et } \\
\text { entraînement de force la plupart des jours de la } \\
\text { semaine } \\
\text { - total d'au moins } 150 \text { min/semaine } \\
\text { gain de poids gestationnel } \\
\text { - programmes de marche ( } 25-40 \text { min) } 3 \text { ou } 4 \\
\text { fois par semaines : diminution de } 50 \% \text { du } \\
\text { gain de poids gestationnel et les besoins } \\
\text { d'insulines ont diminuées puisque la } \\
\text { régulation du glucose est plus efficace } \\
\text { - programmes aquatiques sont prouvé d'être } \\
\text { efficace si les installations y sont } \\
\text { Poids à la naissance } \\
\text { - AP n'augmenta pas les risques de } \\
\text { microsomie } \\
\text { - } 120 \text { min à } 150 \text { min d'AP diminuent les } \\
\text { risques de macrosomie de } 30 \% \\
\text { - Effet plus significatif durant le } 3 \text { e trimestre } \\
\text { Naissance prématurée } \\
\text { - diminue les risques (+ de vascularisation au } \\
\text { placenta et moins de stress oxydatif) }\end{array}$ \\
\hline $\begin{array}{l}\text { 7.Evidence Brief : Risk } \\
\text { Factors for Large for } \\
\text { Gestational Âge (LGA) } \\
\text { Infants in Ontario (Santé } \\
\text { publique Ontario, 2015) }\end{array}$ & $\begin{array}{l}\text { le poids de } \\
\text { naissance est } \\
\text { supérieur au 90e } \\
\text { centile du poids de } \\
\text { naissance spécifique } \\
\text { au sexe pour la } \\
\text { référence d'âge } \\
\text { gestationnel, }\end{array}$ & $\begin{array}{l}\text { Diabète gestationnel } \\
\text { Augmentation des risques } \\
\text { de } 1,59 \% \\
\text { préexistant } \\
\text { Augmentation des risques } \\
\text { de } 4,75 \% \\
\text { Age } \\
\text { Moins que } 17 \text { ans } \\
\text { augmentation des risques }\end{array}$ & $\begin{array}{l}10 \% \text { en } \\
2012-2013\end{array}$ & $\begin{array}{l}\text { - accouchement plus long } \\
\text { - césarienne } \\
\text { - dystocie de l'épouse } \\
\text { - Hypoxie fœetale } \\
\text { - mort intra-utérine } \\
\text { Long terme } \\
\text { - risques de développer le diabète, } \\
\text { l'obésité, l'asthme et le cancer }\end{array}$ & $\begin{array}{l}\text { - intervention en santé publique seraient } \\
\text { importantes pour permettre aux femmes } \\
\text { d'avoir un poids santé et de le maintenir au } \\
\text { cours de la grossesse }\end{array}$ \\
\hline
\end{tabular}


Diversity of Research in Health Journal/ Revue de la Diversité de la Recherche en Santé Vol 4, January/ Janvier 2021 - ISSN: 2561 -1666 DOI : 10.28984/drhj.v4i1.335

\begin{tabular}{|c|c|c|}
\hline & $\begin{array}{l}\text { de } 1,39 \\
\text { IMC } \\
-(>25): 1,59 \% \\
\text { d'augmentation des risques } \\
-(>30) 2,31 \% \\
\text { d'augmentation des risques } \\
\text { Gain de poids gestationnel } \\
\text { Plus que les } \\
\text { recommandations : } \\
\text { augmentation des risques } \\
\text { de } 3 \% \\
\text { Obésité (avant ou durant) } \\
\text { est assise à } 60 \% \text { de tous } \\
\text { les cas de macrosomie de } \\
\text { l'étude } \\
\text { Parité } \\
\text { Augmentation des risques } \\
\text { de } 1,60 \text {-2,24\% quand la } \\
\text { parité est plus que } 0 \\
\text { Historique de naissance } \\
\underline{\text { macrosomique }} \\
\text { Augmentation des risques } \\
\text { de } 4,5 \%\end{array}$ & \\
\hline $\begin{array}{l}\text { 8.Determinants of } \\
\text { Pregnant Women's Online } \\
\text { Self-Regulatory Activities } \\
\text { for Appropriate } \\
\text { Gestational Weight Gain } \\
\text { (Kim, 2015) }\end{array}$ & $\begin{array}{l}\text { Gain de poids gestationnel } \\
\text { excessif } \\
\text { Ethnie : Hispanique, afro- } \\
\text { américain } \\
\text { statut social écologique } \\
\text { plus bas } \\
\text { Autres enfants à la maison }\end{array}$ & $\begin{array}{l}\text { Croyance qu'un gain de poids santé va } \\
\text { entraîner un état de santé pour l'enfant } \\
\text { augmente la possibilité de fixation d'objectifs } \\
\text { et auto surveillance } \\
\text { Ces deux éléments augmentent la possibilité } \\
\text { d'avoir un meilleur contrôle de poids lors de la } \\
\text { grossesse } \\
\text { La grossesse est une opportunité }\end{array}$ \\
\hline
\end{tabular}


Diversity of Research in Health Journal/ Revue de la Diversité de la Recherche en Santé Vol 4, January/ Janvier 2021 - ISSN: 2561 -1666 DOI : 10.28984/drhj.v4i1.335

\begin{tabular}{|c|c|c|}
\hline & (moins de temps) & $\begin{array}{l}\text { d'apprentissage ou il y a une augmentation de } \\
\text { motivation pour adopter des attitudes saines } \\
\text { - nutrition équilibrée et AP régulière : ces } \\
\text { interventions qui font la promotion de } \\
\text { techniques autorégulations sont plus efficaces } \\
\text { Ex } \rightarrow \text { fixation d'objectifs et l'enregistrement de } \\
\text { leurs activités } \\
\text { Utilisation de l'internet pour donner ses outils } \\
\text { et permettre de rejoindre une plus grande } \\
\text { population } \\
\text { Prendre en compte les facteurs externes tels } \\
\text { que les autres enfants à la maison } \\
\text { L'autorégulation de l'AP dépend du degré de } \\
\text { motivation envers le contrôle de soi } \\
\text { L'auto efficacité est la croyance d'une } \\
\text { personne en ses propres habiletés, augmenter } \\
\text { ceci va entraîner un style de de vie plus santé } \\
\text { Commencer avec un diagnostic, ensuite fixer } \\
\text { des buts, nommer les obstacles possibles et les } \\
\text { stratégies pour les surmonter. } \\
\text { Constamment une révision des objectifs }\end{array}$ \\
\hline $\begin{array}{l}\text { 9. Sport et grossesse : une } \\
\text { nécessaire prescription } \\
\text { (Maître, 2013) }\end{array}$ & Prise de poids gestationnel & $\begin{array}{l}\text { Ap pour la prévention du diabète gestationnel } \\
\text { et surpoids } \\
\text { Programme de marche } 3 \mathrm{x} / \text { semaines = moins } \\
\text { d'incidence de macrosomie } \\
\text { 3e trimestre renforcement musculaire des } \\
\text { membres supérieurs est plus facile } \\
\text { Recommendations } \\
-60-70 \% \text { de la FCmax }\end{array}$ \\
\hline
\end{tabular}


Diversity of Research in Health Journal/ Revue de la Diversité de la Recherche en Santé Vol 4, January/ Janvier 2021 - ISSN: 2561 -1666 DOI : 10.28984/drhj.v4i1.335

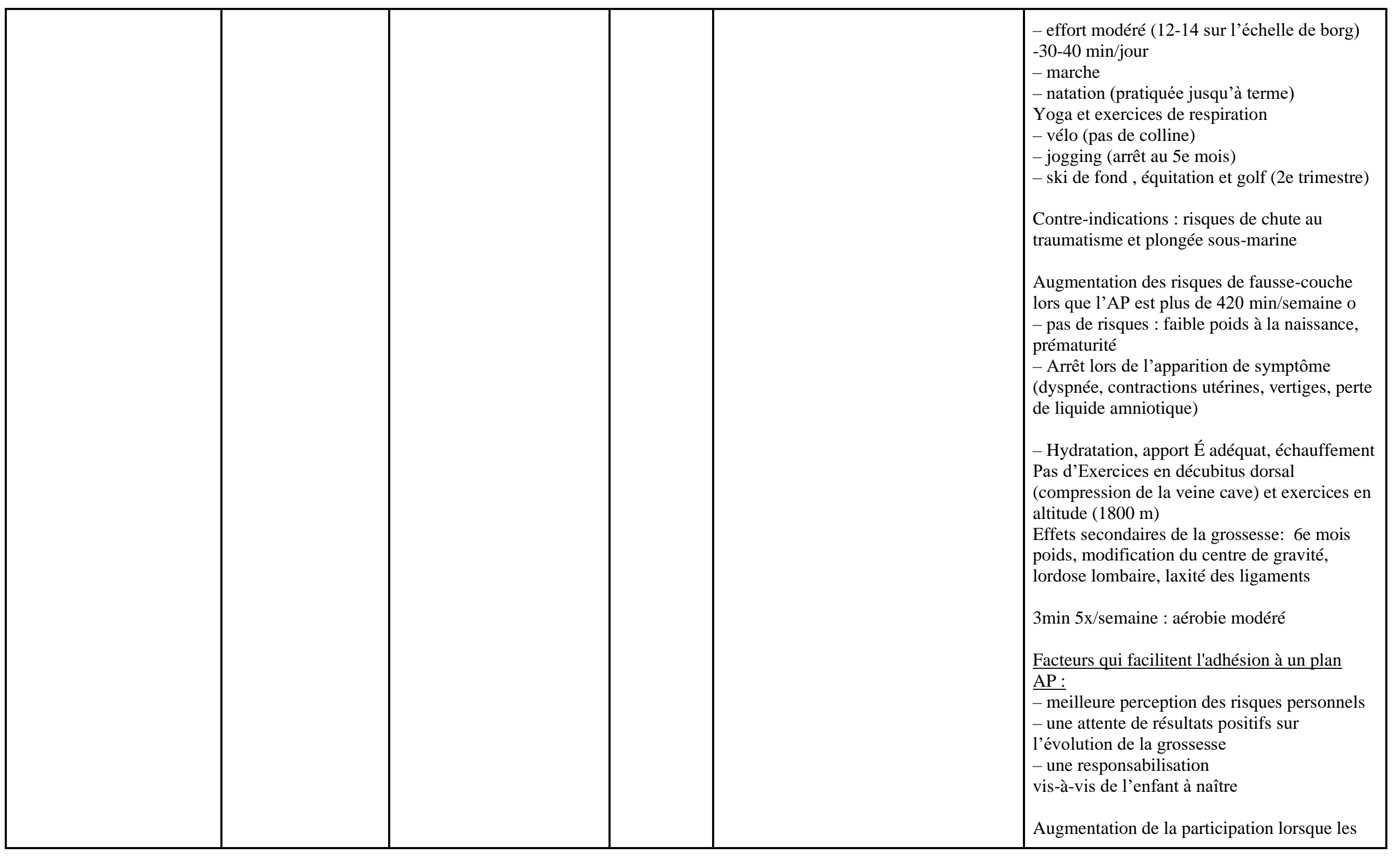


Diversity of Research in Health Journal/ Revue de la Diversité de la Recherche en Santé Vol 4, January/ Janvier 2021 - ISSN: 2561 -1666 DOI : 10.28984/drhj.v4i1.335

\begin{tabular}{|c|c|c|c|c|}
\hline & & & & $\begin{array}{l}\text { activités sont structurées } \\
\text { Adaptations } \mathrm{CV} \text { et respiratoires à la grossesse } \\
\text { sont favorables pour la pratique AP (ex : } \\
\text { augmentation du Volume plastique) }\end{array}$ \\
\hline $\begin{array}{l}\text { 10. Effects of aerobic } \\
\text { exercise training on } \\
\text { maternal and neonatal } \\
\text { outcome: a } \\
\text { randomized controlled } \\
\text { trial on pregnant women } \\
\text { in Iran (Ghodsi, 2014) }\end{array}$ & & & & $\begin{array}{l}\text { American College of Sports: } 30 \mathrm{~min} / \mathrm{jours} \\
\text { d'exercices aérobies } 3 \text { jours ou plus par } \\
\text { semaines (marche, vélo, natation, et autres } \\
\text { exercices sans-impact) } \\
\frac{\text { Étude }}{15 \mathrm{~min} \text { de vélo ( } 50-60 \% \text { de la FC max) } 3} \\
\text { fois/semaines diminue le poids fotal ( } 160 \mathrm{~g} \text { en } \\
\text { moyenne) pour être dans la plage normale }\end{array}$ \\
\hline $\begin{array}{l}\text { 11. The effect of maternal } \\
\text { exercise } \\
\text { during pregnancy on } \\
\text { abnormal } \\
\text { fetal growth } \\
\text { (Tomic, 2013) }\end{array}$ & $>4000 \mathrm{~g}$ & $\begin{array}{l}\text { Statut socio-économique } \\
\text { - statut marital } \\
\text { - pression artérielle } \\
\text { Diabète } \\
\text { Utilisation de la cigarette } \\
\text { Soins prénataux }\end{array}$ & $\begin{array}{l}\text { Hémorragies post-partum } \\
\text { Césarian } \\
\text { Dystocie de l'épaule } \\
\text { Traumatismes à l'accouchement } \\
\text { - risques d'obésité et diabète chez } \\
\text { l'enfant } \\
\text { Conséquences sérieuses à la santé } \\
\text { publique }\end{array}$ & $\begin{array}{l}\text {-30 min 3/semaines } \\
60-70 \% \text { de la FCmax (2,5 MET) } \\
\text { Effet protecteur des risques de macrosomie : } \\
\text { meilleure tolérance au glucose, } \\
\text { Effet plus significatif lorsque l'exercice est } \\
\text { modéré et légère }\end{array}$ \\
\hline
\end{tabular}

Tableau 2 : Césarienne

\begin{tabular}{|l|l|l|l|l|l|}
\hline Titre de l'Article & $\begin{array}{l}\text { Nature } \\
\text { C'est quoi la } \\
\text { césarienne }\end{array}$ & $\begin{array}{l}\text { Étiologie } \\
\text { (causes à la césarienne) }\end{array}$ & Prévalence & $\begin{array}{l}\text { Caractéristiques (Effets de l'AP sur la } \\
\text { césarienne, ou se qui peut lié vers la } \\
\text { césarienne) }\end{array}$ & \begin{tabular}{l} 
intervention \\
\hline $\begin{array}{l}\text { Organisation Mondiale de } \\
\text { la Santé (OMS, 2018) } \\
\text { http://apps.who.int/gho/dat } \\
\text { a/node.main.BIRTHSBYC }\end{array}$
\end{tabular} \\
\hline
\end{tabular}


Diversity of Research in Health Journal/ Revue de la Diversité de la Recherche en Santé Vol 4, January/ Janvier 2021 - ISSN: 2561 -1666 DOI : 10.28984/drhj.v4i1.335

\begin{tabular}{|c|c|c|c|c|}
\hline$\underline{\text { AESAREAN?lang=en }}$ & & & & \\
\hline $\begin{array}{l}\text { Canadian Institute for } \\
\text { Health Information (CIHI, } \\
\text { 2018) } \\
\text { https://www.cihi.ca/en/c- } \\
\text { section-rates-continue-to- } \\
\text { increase-while-birth-rates- } \\
\text { decline }\end{array}$ & & $\begin{array}{l}102 \text { pour } \\
10000 \\
\text { habitants } \\
28.2 \% \\
(2016- \\
2017)\end{array}$ & & \\
\hline $\begin{array}{l}\text { 1.Influence of prenatal } \\
\text { physical activity on the } \\
\text { course of labour and } \\
\text { delivery according to the } \\
\text { Polish standard for } \\
\text { perinatal care } \\
\text { (Szumilewicz et al., 2013) }\end{array}$ & $\begin{array}{l}\text { - Utilisation des } \\
\text { anesthésies et analgésie. } \\
\text { - induction à } \\
\text { l'Accouchement }\end{array}$ & $\begin{array}{l}10-15 \% \\
(\mathrm{OMS}, \\
\text { avant } \\
2013)\end{array}$ & $\begin{array}{l}\text { - Morbidité et la mort, } \\
\text { - problème respiratoire chez l'enfant. }\end{array}$ & $\begin{array}{l}-150 \text { minutes d'AP peuvent réduire les risques } \\
\text { de césarienne. } \\
\text { - AP peut développer une mentalité de } \\
\text { capabilité ("can do" mentality) à ne pas avoir } \\
\text { une césarienne. } \\
\text { Test : } \\
-845 \text { femmes }(6,7 \% \text { césarienne avec grande } \\
\text { intensité d'exercice) }(28,1 \% \text { sédentaire) } \\
\text { (HALL et KAUFMANN) } \\
-137 \text { premières grossesses, AP = moins de } \\
\text { risque à la césarienne. } 4.5 \text { fois plus relative } \\
\text { pour sédentaire (n=93) que pour les femmes } \\
\text { active (n-44) (BUNGUM et AL.) }\end{array}$ \\
\hline $\begin{array}{l}\text { 2.Effect of physical } \\
\text { activity during pregnancy } \\
\text { on mode of delivery } \\
\text { (Domenjoz, et al., 2014) }\end{array}$ & $\begin{array}{l}\text { - Réduire le poids à la } \\
\text { naissance qui réduit les } \\
\text { chances de césarienne } \\
\text { - IMC (augmentation de } \\
\text { césarienne on une } \\
\text { corrélation avec } \\
\text { augmentation d'IMC }\end{array}$ & $\begin{array}{l}\text { - augmente } \\
\text { durant la } \\
\text { dernière } \\
\text { décennie. }\end{array}$ & $\begin{array}{l}\text { - Morbidité et mort pour enfant et } \\
\text { mère. } \\
\text { - Risque à court terme = infection, } \\
\text { thromboembolie et hémorragie sévères } \\
\text { à temps. } \\
\text { - Morbidité long terme = rupture } \\
\text { utérine }\end{array}$ & $\begin{array}{l}\text { - Exercice régulier réduit le risque de } \\
\text { césarienne. } \\
\text { - Exercice à résistance et aérobique réduisent } \\
\text { le risque de césarienne de } 15 \% \text { et réduisent le } \\
\text { gain maternel de poids par } 1 \mathrm{~kg} \text { en moyenne. } \\
\text { - Même les femmes avec moins d'heures } \\
\text { d'exercice contribuent à réduire les } \\
\text { césariennes. } \\
\text { - Primipare } 3 \text { fois plus de chance à avoir une } \\
\text { césarienne qu'une femme multipare. }\end{array}$ \\
\hline 3.Bénéfices et risques & - rupture utérine & & - morbidité maternelle infectieuse et & $-90 \%$ des femmes (É-U) qui ont accouché \\
\hline
\end{tabular}


Diversity of Research in Health Journal/ Revue de la Diversité de la Recherche en Santé Vol 4, January/ Janvier 2021 - ISSN: 2561 -1666 DOI : 10.28984/drhj.v4i1.335

\begin{tabular}{|c|c|c|c|c|c|}
\hline $\begin{array}{l}\text { maternels de la tentative } \\
\text { de voie basse comparée à } \\
\text { la césarienne programmée } \\
\text { en cas d'antécédent de } \\
\text { césarienne (Beucher et al., } \\
\text { 2012) }\end{array}$ & & $\begin{array}{l}\text { - signes cliniques non } \\
\text { spécifiques (anomalies du } \\
\text { rythme cardiaque (FC) } \\
\text { fœetal, altération de la } \\
\text { progression du travail, } \\
\text { douleur ou hémorragie } \\
\text { maternelle.) } \\
\text { - échec d'un travail voie } \\
\text { basse (accouchement } \\
\text { normal) }\end{array}$ & & $\begin{array}{l}\text { hémorragique. } \\
\text { - mortalité maternelle (rare) } \\
\text { - augmente le risque de complications } \\
\text { opératoire (plaie de vessie, plaie } \\
\text { d'artère utérine, plaie digestive, plaie } \\
\text { de l'uretère, déchirure du ligament } \\
\text { large). }\end{array}$ & $\begin{array}{l}\text { avant en césarienne-bénéfice d'une césarienne } \\
\text { programmée. } \\
\text { - prévention des ruptures utérines }\end{array}$ \\
\hline $\begin{array}{l}\text { 4.Caesarean section versus } \\
\text { vaginal delivery for } \\
\text { preterm birth in singletons } \\
\text { (Review) (Alfirevic et al., } \\
\text { 2013) }\end{array}$ & & & & $\begin{array}{l}\text { - risque accru de morbidité respiratoire } \\
\text { chez le nouveau-né. } \\
\text { - Césarienne vs voie basse : } 3 \text { à } 5 \text { fois } \\
\text { plus haut pour la mortalité maternelle, } \\
2 \text { fois plus haute pour hystérectomie, } 2 \\
\text { fois plus haut et un montant de temps à } \\
\text { l'hôpital plus long. } \\
\text { - Infection, clos sanguins, embolie } \\
\text { pulmonaire, perte sanguine excessive = } \\
\text { césarienne. }\end{array}$ & $\begin{array}{l}\text { - clos veineux profond, choc endotoxique, } \\
\text { septicémie puerpérale et rupture de la plaie } \\
\text { (Maternelle) }\end{array}$ \\
\hline $\begin{array}{l}\text { 5.Safe prevention of the } \\
\text { primary cesarean delivery } \\
\text { (American College of } \\
\text { Obstetricians and } \\
\text { Gynecologists (the } \\
\text { College et coll.) and the } \\
\text { Society for Maternal-Fetal } \\
\text { Medicine, 2014) }\end{array}$ & & $\begin{array}{l}\text { - difficulté du travail } \\
\text { (labor) } \\
\text { - suivi anormal ou } \\
\text { indéterminé de la FC du } \\
\text { fœetus } \\
\text { - malorientation du fétu. } \\
\text { - suspicion de macrosomie } \\
\text { - gestation multiple } \\
\text { - induction du travail }\end{array}$ & $\begin{array}{l}-2011(E ́- \\
\text { U) } 1 \text { sur } 3 \\
\text { des } \\
\text { naissances } \\
\text { était par } \\
\text { césarienne }\end{array}$ & $\begin{array}{l}\text { - morbidité maternelle } 9.2 \% \text {, } \\
\text { - mortalité maternelle } 2.7 \% \\
\text { - risque de complications } 3 \text { fois plus : } \\
\text { hystérectomie, hémorragie, } \\
\text { complication anesthésique, rupture } \\
\text { utérine, etc. } \\
\text { - contrairement, induction devrait être } \\
\text { utilisé afin de réduire le risque de } \\
\text { césarienne. }\end{array}$ & \\
\hline $\begin{array}{l}\text {-Oxford dictionary: } \\
\text { Concise Medical } \\
\text { Dictionary (2010) } \\
\text {-Mayo Clinic (2018) } \\
\text {-Merriam-Webster }\end{array}$ & $\begin{array}{l}\text {-a surgical } \\
\text { operation for } \\
\text { delivering a baby } \\
\text { through the } \\
\text { abdominal wall } \\
\text {-is a surgical } \\
\text { procedure used to }\end{array}$ & & & & \\
\hline
\end{tabular}


Diversity of Research in Health Journal/ Revue de la Diversité de la Recherche en Santé Vol 4, January/ Janvier 2021 - ISSN: 2561 -1666 DOI : 10.28984/drhj.v4i1.335

\begin{tabular}{|c|c|c|c|c|c|}
\hline $\begin{array}{l}\text { Medical Dictionary (2020) } \\
\text { (trois dictionnaires en } \\
\text { ligne reconnue pour le } \\
\text { côté médical) }\end{array}$ & $\begin{array}{l}\text { deliver a baby } \\
\text { through incisions in } \\
\text { the abdomen and } \\
\text { uterus. }\end{array}$ & & & & \\
\hline $\begin{array}{l}\text { 6.Exercise during } \\
\text { pregnancy reduces the rate } \\
\text { of cesarean and } \\
\text { instrumental deliveries: } \\
\text { results of a randomized } \\
\text { controlled trial (Barakat et } \\
\text { al., 2012) }\end{array}$ & & & $\begin{array}{l}+ \text { que } 31 \% \\
\text { mondiale }\end{array}$ & $\begin{array}{l}\text { - Chirurgie abdominale, problème du } \\
\text { placenta pour accouchement futur, } \\
\text { difficulté respiratoire au nouveau-né, } \\
\text { réflexe affaibli de succion chez le } \\
\text { nouveau-né, infection, perte sanguine } \\
\text { excessive, complication respiratoire } \\
\text { maternelle, réaction d'anesthésie et } \\
\text { hospitalisation plus longue. }\end{array}$ & $\begin{array}{l}\text { Intervention = } 3 \text { séances de } 40-45 \text { minutes par } \\
\text { semaine. Utilisation de moniteur de FC. } \\
\text { Intensité légère à modérée (sous } 70 \% \text { de } \\
\text { FCmax, calculer par l'âge). } \\
\text { - \% réduit de césarienne dans le group } \\
\text { d'exercice avec gain de poids excessif } \\
\text { - IMC et gain de poids corporel de manière } \\
\text { propre est en relation avec l'habileté de donner } \\
\text { naissance sans césarienne. } \\
\text { - femme qui continue à faire de l'exercice, } \\
\text { plus petite incidence à la césarienne (6 \% vs } \\
30 \% \text { ) } \\
\text { - femme sédentaire était plus probable à avoir } \\
\text { une césarienne ( } 2,05 \text { fois plus). }\end{array}$ \\
\hline $\begin{array}{l}\text { 7.Associations between } \\
\text { Prenatal Physical Activity } \\
\text { and Neonatal and } \\
\text { Obstetric Outcomes-A } \\
\text { Secondary Analysis of the } \\
\text { Cluster-Randomized } \\
\text { GeliS Trial. (Hoffman et } \\
\text { al., 2019) }\end{array}$ & & & & & $\begin{array}{l}\text { - Femme qui pratique AP lors des } 2 \text { derniers } \\
\text { trimestres, plus de chances à faire } \\
\text { l'accouchement vaginal qui la césarienne. } \\
\text { Aucune différence dans l'incidence de la } \\
\text { césarienne lors de la grossesse entre les } \\
\text { femmes active et inactive. }\end{array}$ \\
\hline $\begin{array}{l}\text { 8.Mode of Delivery } \\
\text { according to Leisure Time } \\
\text { Physical Activity before } \\
\text { and during Pregnancy: A } \\
\text { Multicenter Cohort Study } \\
\text { of Low-Risk Women }\end{array}$ & & $\begin{array}{l}\text { - Obèse ou en surpoids } \\
\text { - âge }\end{array}$ & & $\begin{array}{l}\text { - risque de complication d'anesthésie, } \\
\text { perte sanguine excessive, complication } \\
\text { respiratoire, temps récupération plus } \\
\text { long, etc. (maternelle) }\end{array}$ & $\begin{array}{l}\text { - Plus haut le niveau d'AP, moins les chances } \\
\text { de césarienne urgent. } \\
\text { - Femme active moins probable d'avoir un } \\
\text { accouchement compliqué. }\end{array}$ \\
\hline
\end{tabular}


Diversity of Research in Health Journal/ Revue de la Diversité de la Recherche en Santé Vol 4, January/ Janvier 2021 - ISSN: 2561 -1666 DOI : 10.28984/drhj.v4i1.335

\begin{tabular}{|c|c|c|c|}
\hline (Neilsen, 2017) & & & \\
\hline $\begin{array}{l}\text { 9.Physical Activity and } \\
\text { Excess Weight in } \\
\text { Pregnancy Have } \\
\text { Independent and Unique } \\
\text { Effects on Delivery and } \\
\text { Perinatal Outcomes } \\
\text { (Morgan, 2014) }\end{array}$ & $\begin{array}{l}\text { - IMC } \\
\text { - Surpoid et obésité }\end{array}$ & - risque pour la mère et l'enfant. & $\begin{array}{l}\text { - } 23 \% \text { césarienne } \\
\text { - tableau } 2 \text { montre que l'AP de haute intensité } \\
\text { = moins de césariennes comparées à l'AP } \\
\text { basse intensité } \\
\text { - aucune relation entre IMC et césarienne } \\
\text { - L'AP taux diminué de césarienne } \\
\text { - Augmentation du niveau d'AP réduit le } \\
\text { nombre de césariennes }\end{array}$ \\
\hline $\begin{array}{l}\text { 10.Exercise during } \\
\text { Pregnancy and Risk of } \\
\text { Late Preterm } \\
\text { Birth,Cesarean Delivery, } \\
\text { and Hospitalizations } \\
\text { (Tinloy, 2013) }\end{array}$ & âge & & $\begin{array}{l}-150 \text { minutes d'AP d'intensité modérée par } \\
\text { semaine. } \\
-29 \% \text { césarienne } \\
(1962 \text {, Erdelyi) a trouvé une réduction de } \\
50 \% \text { de risque de césarienne envers les } \\
\text { athlètes hongroises en contraire à les non- } \\
\text { athlètes } \\
\text { - moins de césariennes chez les femmes qui } \\
\text { font de l'exercice pour } 150 \text { minutes par } \\
\text { semaine comparée aux femmes qui en font } \\
\text { moins. }\end{array}$ \\
\hline
\end{tabular}

Tableau 3 : Déchirure périnéales

\begin{tabular}{|l|l|l|l|l|l|}
\hline $\begin{array}{l}\text { Handicap : Les } \\
\text { déchirures périnéales }\end{array}$ & $\begin{array}{l}\text { Nature } \\
\text { Qu'est-ce que c'est } \\
\text { la déchirure } \\
\text { périnéale? } \\
\text { Comment se } \\
\text { définit-elle? }\end{array}$ & $\begin{array}{l}\text { (Cause de la pathologie) } \\
\text { Prévalence }\end{array}$ & $\begin{array}{l}\text { Caractéristiques } \\
\text { Comment la déchirure périnéale se } \\
\text { manifeste-t-elle? }\end{array}$ & (intervention) \\
\hline
\end{tabular}


Diversity of Research in Health Journal/ Revue de la Diversité de la Recherche en Santé Vol 4, January/ Janvier 2021 - ISSN: 2561 -1666 DOI : 10.28984/drhj.v4i1.335

\begin{tabular}{|c|c|c|c|c|}
\hline $\begin{array}{l}\text { 1. Analyse de l'activité } \\
\text { physique chez la femme } \\
\text { enceinte sportive. (De } \\
\text { Bourg, \& Bresse, 2015) }\end{array}$ & & & & $\begin{array}{l}\text { - Les femmes ayant subi une déchirure } \\
\text { complète du périnée présentent un risque accru } \\
\text { d'incontinence fécale persistante. }\end{array}$ \\
\hline $\begin{array}{l}\text { 2. Symposium } \\
\text { d'obstétrique.(Borracce } \\
\text { tti, 2016) }\end{array}$ & $\begin{array}{l}\text { - L'accouchement mène à } \\
\text { un étirement du périnée } \\
\text { jusqu'à la limite de } \\
\text { l'élasticité qui peut mener } \\
\text { à une déchirure périnéale et } \\
\text { diminue le pouvoir de } \\
\text { contraction des muscles } \\
\text { qui le compose. }\end{array}$ & $\begin{array}{l}\text { - Les } \\
\text { douleurs : } \\
\text { conséquence } \\
\text { immédiate et } \\
\text { fréquente des } \\
\text { délabrements } \\
\text { ci - dessus. } \\
42 \% \text { des } \\
\text { femmes en } \\
\text { post-partum } \\
\text { immédiat, } \\
22 \% \text { après } 8 \\
\text { semaines, } \\
10 \% \text { après } 1 \\
\text { an, surtout } \\
\text { chez les } \\
\text { primipares. } \\
\text { - Troubles } \\
\text { de la } \\
\text { continence } \\
\text { urinaire : } \\
\text { toutes études } \\
\text { confondues, } \\
\text { la } \\
\text { prévalence } \\
\text { de } \\
\text { l'incontiance } \\
\text { urinaire à } 2 \\
\text { mois post- } \\
\text { partum varie } \\
\text { de } 15,5 \text { à } \\
42 \% \text {. De }\end{array}$ & $\begin{array}{l}\text { - Types de déchirures : épisiotomie } \\
\text { (procédure médicale), déchirures } \\
\text { fermées, déchirures ouvertes, brèches } \\
\text { vaginales, fistules obstétricales, } \\
\text { élongations, lésions nerveuses. } \\
\text { - Conséquance : statique pelvienne, } \\
\text { sexualité, incontinence urinaire ou } \\
\text { fécale, image de soi, douleur } \\
\text { - Déficiences musculaires : } \\
\text { diminution de la force musculaire } \\
\text { jusqu'à } 6 \text { semaines max. (post- } \\
\text { partum) } \\
\text { - conséquence d'une hypotonie } \\
\text { musculaire qui laisse la vulve et/ou le } \\
\text { vagin ouvert peut entraîner des } \\
\text { troubles du plaisir et de la gêne } \\
\text { (bruits d'air) } \\
\text { - les femmes avec une déchirure } \\
\text { complète du périnée présentent un } \\
\text { risque accru d'incontinence fécale } \\
\text { persistante. }\end{array}$ & $\begin{array}{l}\text { - La gymnastique prénatale est un moyen } \\
\text { efficace pour éviter des complications à } \\
\text { l'accouchement. } \\
\text { - Plus le nombre de séances augmente, moins } \\
\text { on observe de déchirures du périnée lors d'un } \\
\text { accouchement par voie basse. } \\
\text { - Reprise de l'AP intégré travail du plancher } \\
\text { pelvien et sangle abdominale. } \\
\text { - Ne pas faire des exercices abdominaux qui } \\
\text { ont une pression vers le bas. }\end{array}$ \\
\hline
\end{tabular}


Diversity of Research in Health Journal/ Revue de la Diversité de la Recherche en Santé Vol 4, January/ Janvier 2021 - ISSN: 2561 -1666 DOI : 10.28984/drhj.v4i1.335

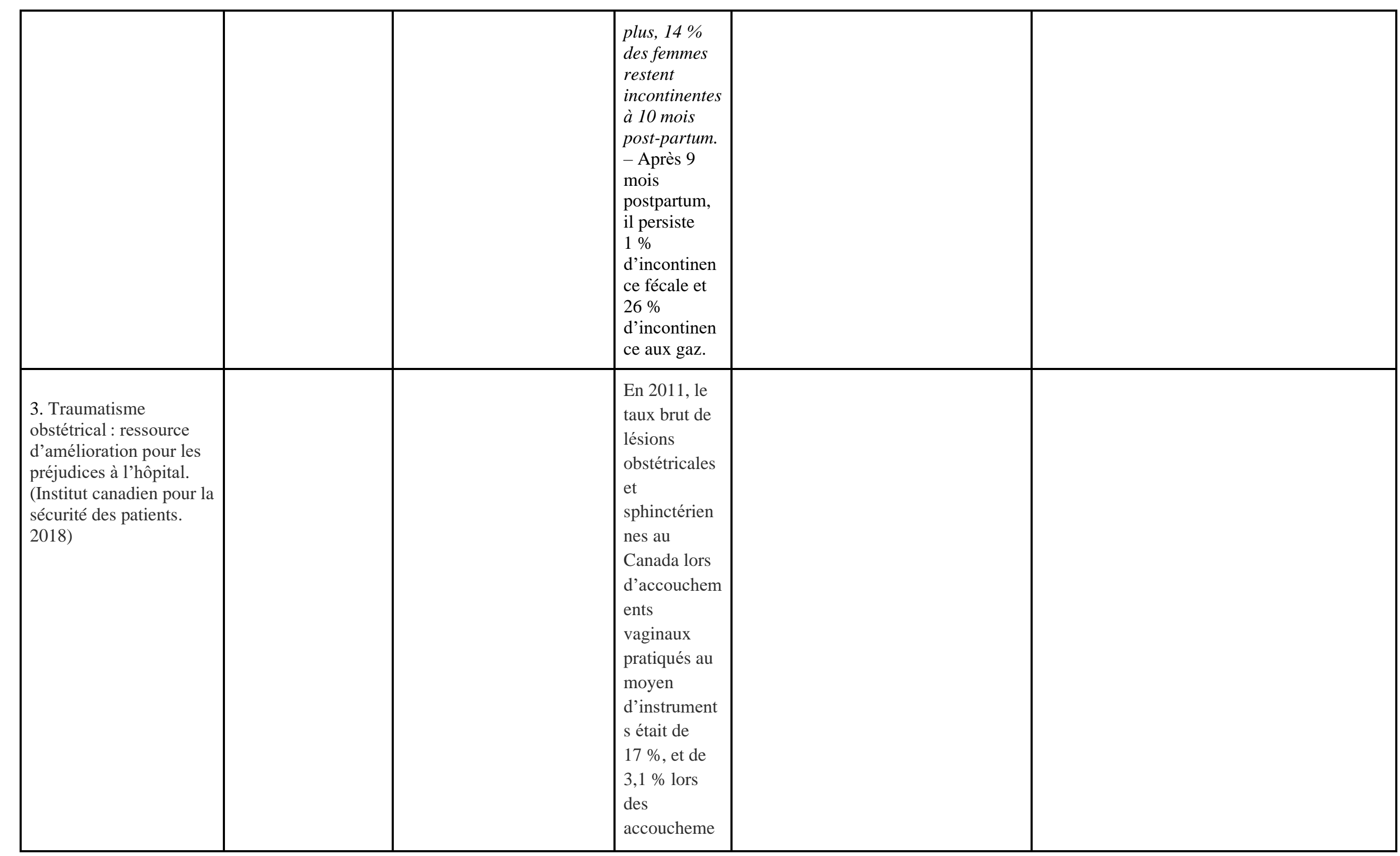


Diversity of Research in Health Journal/ Revue de la Diversité de la Recherche en Santé Vol 4, January/ Janvier 2021 - ISSN: 2561 -1666 DOI : 10.28984/drhj.v4i1.335

\begin{tabular}{|c|c|c|c|c|}
\hline & & $\begin{array}{l}\text { nts vaginaux } \\
\text { pratiqués } \\
\text { sans } \\
\text { instrument } \\
\text { (OCDE, } \\
2013 \text { ). }\end{array}$ & & \\
\hline $\begin{array}{l}\text { 4. Activité physique } \\
\text { chez la femme enceinte : } \\
\text { représentations et } \\
\text { pratiques } \\
\text { professionnelles des } \\
\text { sages-femmes de Nice. } \\
\text { (Carini, 2017) }\end{array}$ & $\begin{array}{l}\text { - Les femmes sportives ont } \\
\text { le périnée très solide, pour } \\
\text { l'accouchement c'est } \\
\text { compliqué, parce que le } \\
\text { périnée n'est pas élastique. }\end{array}$ & & & $\begin{array}{l}\text { - La césarienne et l'utilisation d'instrument } \\
\text { peuvent permettre de réduire le taux de } \\
\text { déchirures périnéales. } \\
\text { - AP important pour garder une bonne sangle } \\
\text { abdominale, pour pousser. } \\
\text { - AP a un effet protecteur sur le périnée. } \\
\text { - Les déchirures périnéales sont un risque, } \\
\text { avec l'AP qui rend le périnée plus tonique. } \\
\text { - La littérature démontre l'effet protecteur de } \\
\text { l'AP sur le périnée. } \\
\text { - la gymnastique prénatale est un moyen } \\
\text { efficace pour éviter des complications à } \\
\text { l'accouchement. } \\
\text { - Plus le nombre de séances augmente, moins } \\
\text { on observe de déchirures du périnée lors d'un } \\
\text { accouchement. }\end{array}$ \\
\hline $\begin{array}{l}\text { 5. Périnée et grossesse. } \\
\text { (Fritel, 2010) }\end{array}$ & $\begin{array}{l}\text { - Les accouchements } \\
\text { laborieux, ou la } \\
\text { maladresse du chirurgien, } \\
\text { en sont les causes les plus } \\
\text { fréquentes des déchirures } \\
\text { périnéales. }\end{array}$ & $\begin{array}{l}\text { Le risque } \\
\text { cumulé de } \\
\text { chirurgie de } \\
\text { la statique } \\
\text { pelvienne est } \\
\text { estimé à } \\
11 \% \text { chez la } \\
\text { femme et } \\
30 \% \text { des } \\
\text { opérées }\end{array}$ & $\begin{array}{l}\text { - L'incontinence urinaire est un } \\
\text { résultat des déchirures. } \\
\text { - L'accouchement vaginal est } \\
\text { susceptible de provoquer des lésions } \\
\text { du nerf pudendal, du sphincter anal } \\
\text { ou des releveurs de l'anus. }\end{array}$ & $\begin{array}{l}\text { Lien entre ces lésions et les symptômes } \\
\text { périnéaux on ignore quels pourraient être les } \\
\text { moyens de prévention. }\end{array}$ \\
\hline
\end{tabular}


Diversity of Research in Health Journal/ Revue de la Diversité de la Recherche en Santé Vol 4, January/ Janvier 2021 - ISSN: 2561 -1666 DOI : 10.28984/drhj.v4i1.335

\begin{tabular}{|c|c|c|c|c|}
\hline & & & $\begin{array}{l}\text { nécessitent } \\
\text { une } \\
\text { deuxième } \\
\text { intervention } \\
\text { (déchirure/d } \\
\text { ommage au } \\
\text { nerfs) }\end{array}$ & \\
\hline $\begin{array}{l}\text { 6. Définitions, } \\
\text { épidémiologie et } \\
\text { facteurs de risque des } \\
\text { lésions périnéales du } 3 \mathrm{e} \\
\text { et } 4 \mathrm{e} \text { degrés. RPC } \\
\text { Prévention et protection } \\
\text { périnéale en obstétrique } \\
\text { CNGOF. (Thubert et al., } \\
2018 \text { ) }\end{array}$ & $\begin{array}{l}\text { - Déchirures } \\
\text { obstétricales du } \\
\text { sphincter de l'anus, } \\
\text { utilisé l'acronyme } \\
\text { LOSA (lésions } \\
\text { obstétricales du } \\
\text { sphincter de l'anus) } \\
\text { au lieu des termes } \\
\text { de «périnée } \\
\text { complet» }\end{array}$ & $\begin{array}{l}\text { - Facteurs associés à la } \\
\text { LOSA : la nulliparité } \\
\text { (jamais accoucher avant), } \\
\text { l'accouchement } \\
\text { instrumental, l'âge } \\
\text { maternel élevé, } \\
\text { l'antécédent de LOSA, la } \\
\text { macrosomie, l'épisiotomie, } \\
\text { la présentation céphalique } \\
\text { en variété postérieure, et } \\
\text { un travail long. }\end{array}$ & $\begin{array}{l}\text { - La } \\
\text { prévalence } \\
\text { des LOSA } \\
\text { types } \\
\text { confondus } \\
\text { est entre } 0,25 \\
\text { à } 6 \% \text {. } \\
\text { - Lors } \\
\text { d'antécédent } \\
\text { de LOSA, la } \\
\text { prévalence } \\
\text { des récidives } \\
\text { se trouve } \\
\text { entre } 5,1 \text { et } \\
10,7 \% \text { à } \\
\text { l'accouchem } \\
\text { ent suivant. }\end{array}$ & $\begin{array}{l}\text { - La formation et la sensibilisation des } \\
\text { praticiens de la naissance au diagnostic de } \\
\text { LOSA améliorent la détection des LOSA en } \\
\text { salle de naissance }\end{array}$ \\
\hline $\begin{array}{l}\text { 7. Mode de poussée à } \\
\text { l'accouchement et } \\
\text { conséquences pelviennes } \\
\text { périnéales. (Ratier, } \\
2015 \text { ) }\end{array}$ & & $\begin{array}{l}\text { - Type de poussées à } \\
\text { l'accouchement soit } \\
\text { Valsalva (expiration } \\
\text { bouche et nez bloqués) et } \\
\text { par expiration. }\end{array}$ & & $\begin{array}{l}\text { - Les deux techniques de poussée lors de } \\
\text { l'accouchement sont comparables en termes } \\
\text { de durée, de risque de déchirure périnéale et } \\
\text { d'état néonatal à la naissance. }\end{array}$ \\
\hline 8. Prévention et & $\begin{array}{l}\text { Déchirures } \\
\text { obstétricales du }\end{array}$ & & & - Accouchement normal, l'épisiotomie n'est \\
\hline
\end{tabular}


Diversity of Research in Health Journal/ Revue de la Diversité de la Recherche en Santé Vol 4, January/ Janvier 2021 - ISSN: 2561 -1666 DOI : 10.28984/drhj.v4i1.335

\begin{tabular}{|c|c|c|c|c|c|}
\hline $\begin{array}{l}\text { protection périnéale en } \\
\text { obstétrique : } \\
\text { Recommandations pour } \\
\text { la Pratique Clinique du } \\
\text { CNGOF. (Ducarme et } \\
\text { al., 2018) }\end{array}$ & $\begin{array}{l}\text { sphincter de l'anus, } \\
\text { utilisé l'acronyme } \\
\text { LOSA (lésions } \\
\text { obstétricales du } \\
\text { sphincter de l'anus) } \\
\text { au lieu des termes } \\
\text { de «périnée } \\
\text { complet» } \\
\text { - Il existe } 4 \text { degrés } \\
\text { de gravité. }\end{array}$ & & & & $\begin{array}{l}\text { pas recommandée pour réduire le risque de } \\
\text { LOSA. } \\
\text { - Accouchement instrumental, une } \\
\text { épisiotomie peut être recommandée pour } \\
\text { éviter une LOSA. } \\
\text { - Il n'est pas recommandé de proposer une } \\
\text { césarienne programmée comme prévention de } \\
\text { lésions ou de dysfonctions périnéales. } \\
\text { - Prévention des LOSA, il n'est pas } \\
\text { recommandé de pratiquer des exercices pour } \\
\text { renforcer les muscles du plancher pelvien } \\
\text { pendant la grossesse. }\end{array}$ \\
\hline $\begin{array}{l}\text { 9. Effect of physical } \\
\text { activity during } \\
\text { pregnancy on mode of } \\
\text { delivery. (Domenjoz et } \\
\text { al., 2014) }\end{array}$ & & & & & $\begin{array}{l}\text { - Avec le manque de résultats, il n'a pas été } \\
\text { possible de prouver que l'AP réduit le risque } \\
\text { de déchirures périnéales. Mais toutes les } \\
\text { femmes qui n'ont pas de complication pendant } \\
\text { la grossesse bénéficient de l'AP. }\end{array}$ \\
\hline $\begin{array}{l}\text { 10. Influence of } \\
\text { prenatal physical } \\
\text { activity on the course of } \\
\text { labour and delivery } \\
\text { according to the new } \\
\text { Polish standard for } \\
\text { perinatal care. } \\
\text { (Szumilewicz et al., } \\
\text { 2013) }\end{array}$ & $\begin{array}{l}\text { - Un trauma } \\
\text { périnéal est défini } \\
\text { comme n'importe } \\
\text { quel dommage } \\
\text { génital pendant } \\
\text { l'accouchement qui } \\
\text { a lieu naturellement } \\
\text { ou par épisiotomie. }\end{array}$ & $\begin{array}{l}\text { - L'accouchement naturel } \\
\text { - Association entre } \\
\text { l'inactivité physique } \\
\text { pendant la période pré } \\
\text { gestationnel (peut être } \\
\text { biaisé par les auteurs) } \\
\text { - le niveau faible d'AP de } \\
\text { la mère réduit sa capacité } \\
\text { de pousser ce qui } \\
\text { augmente le temps } \\
\text { d'accouchement et est } \\
\text { associé avec des taux plus }\end{array}$ & $\begin{array}{l}-85 \% \text { des } \\
\text { femmes au } \\
\text { nord de } \\
\text { l'Irlande } \\
\text { vont } \\
\text { maintenir } \\
\text { des } \\
\text { problèmes en } \\
\text { lien avec } \\
\text { leur trauma } \\
\text { périnéal. } \\
\text { - Les taux de }\end{array}$ & $\begin{array}{l}\text { Peut causer des problèmes } \\
\text { d'incontinence urinaire et/ou fécale. }\end{array}$ & $\begin{array}{l}\text { - Ne supporte pas l'idée que l'AP et les } \\
\text { exercices du plancher pelvien peuvent } \\
\text { augmenter la résistance des muscles périnéaux } \\
\text { et d'avoir un travail plus long lors de } \\
\text { l'accouchement. } \\
\text { - Besoin de trouver une intervention qui } \\
\text { diminue le taux d'épisiotomie et de déchirures } \\
\text { périnéales. } \\
\text { - Des sessions d'exercices pour les femmes } \\
\text { enceintes diminuent la prévalence de déchirure } \\
\text { périnéale. } \\
\text { - L'entraînement n'a pas d'effet sur les }\end{array}$ \\
\hline
\end{tabular}


Diversity of Research in Health Journal/ Revue de la Diversité de la Recherche en Santé Vol 4, January/ Janvier 2021 - ISSN: 2561 -1666 DOI : 10.28984/drhj.v4i1.335

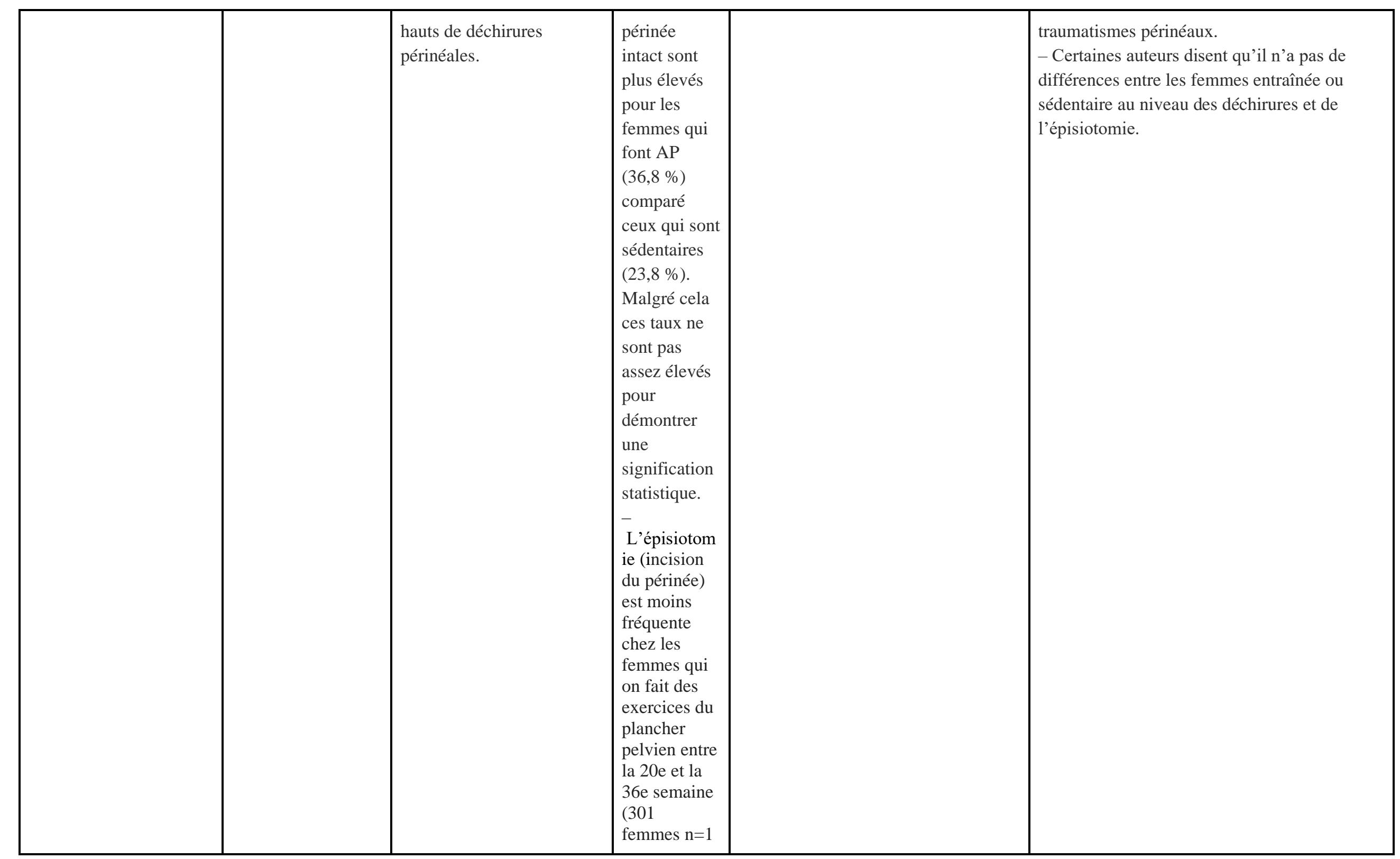


Diversity of Research in Health Journal/ Revue de la Diversité de la Recherche en Santé Vol 4, January/ Janvier 2021 - ISSN: 2561 -1666 DOI : 10.28984/drhj.v4i1.335

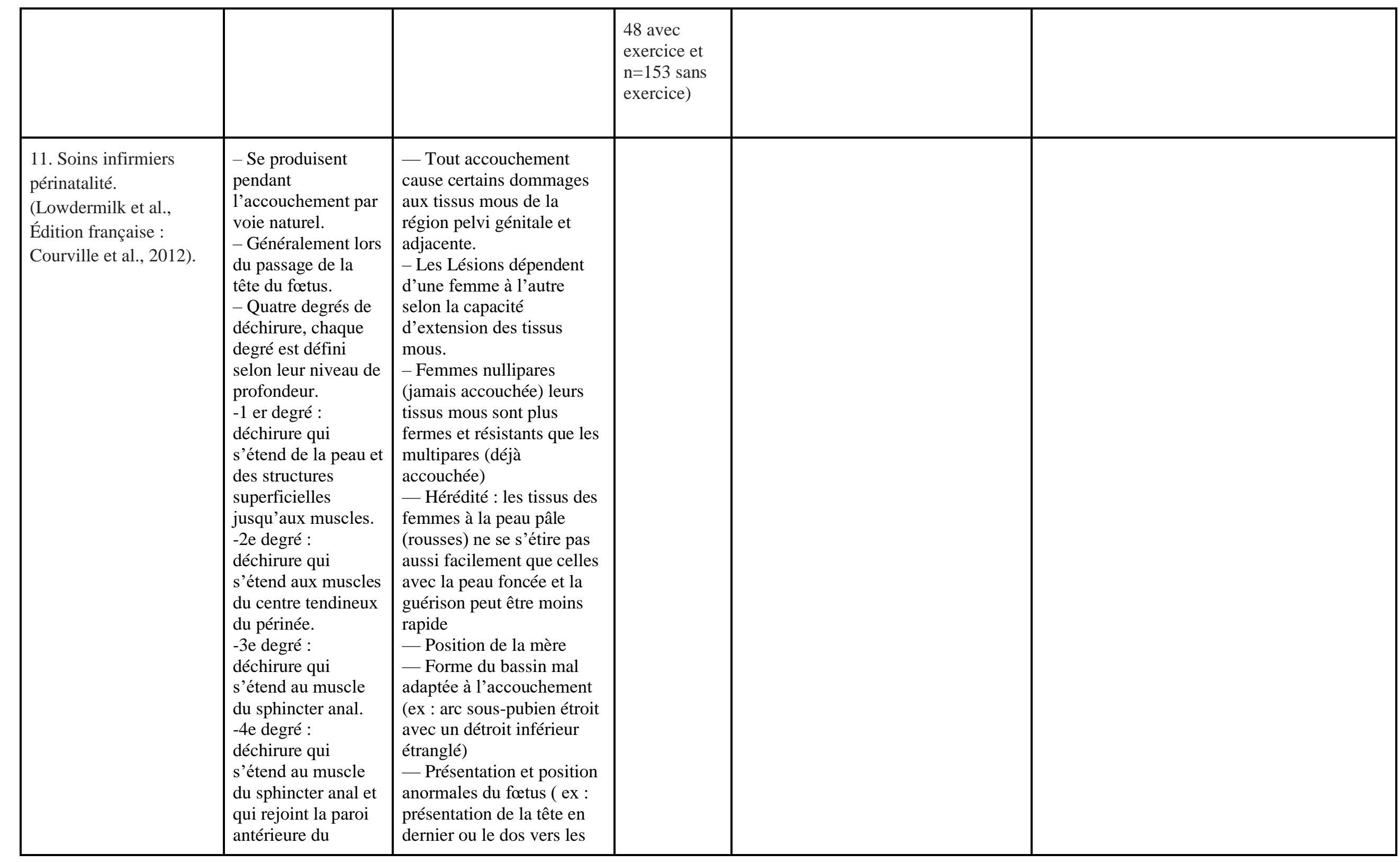


Diversity of Research in Health Journal/ Revue de la Diversité de la Recherche en Santé Vol 4, January/ Janvier 2021 - ISSN: 2561 -1666 DOI : 10.28984/drhj.v4i1.335

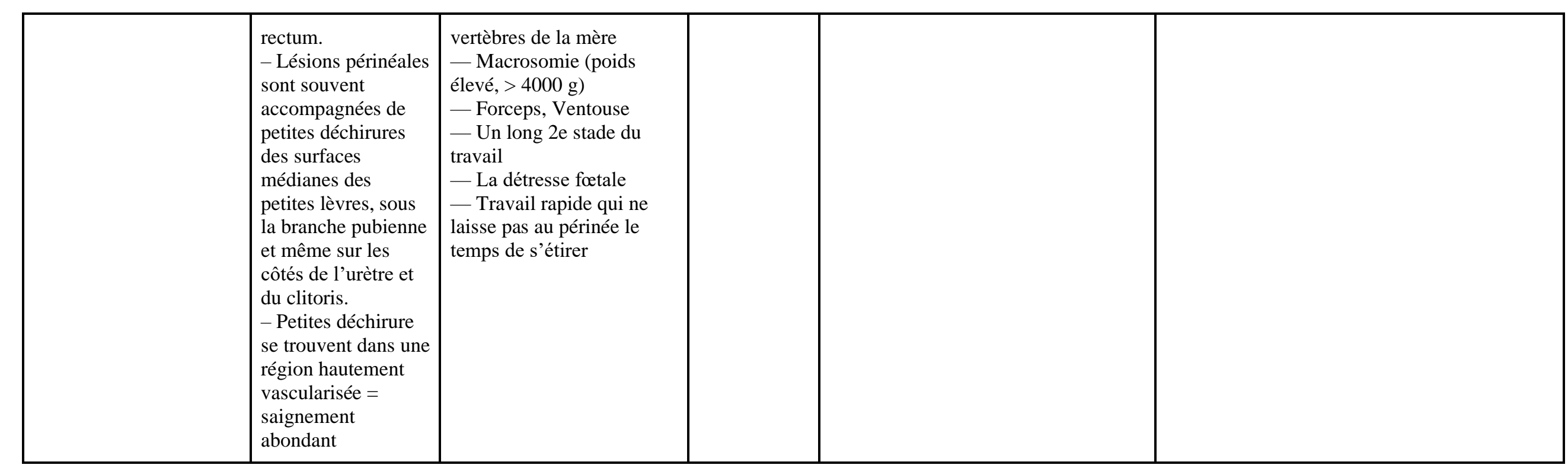


Diversity of Research in Health Journal/ Revue de la Diversité de la Recherche en Santé Vol 4, January/ Janvier 2021 - ISSN: 2561 -1666 DOI : 10.28984/drhj.v4i1.335

Annexe 2

$\underline{\text { Schéma de la corrélation entre les variables }}$

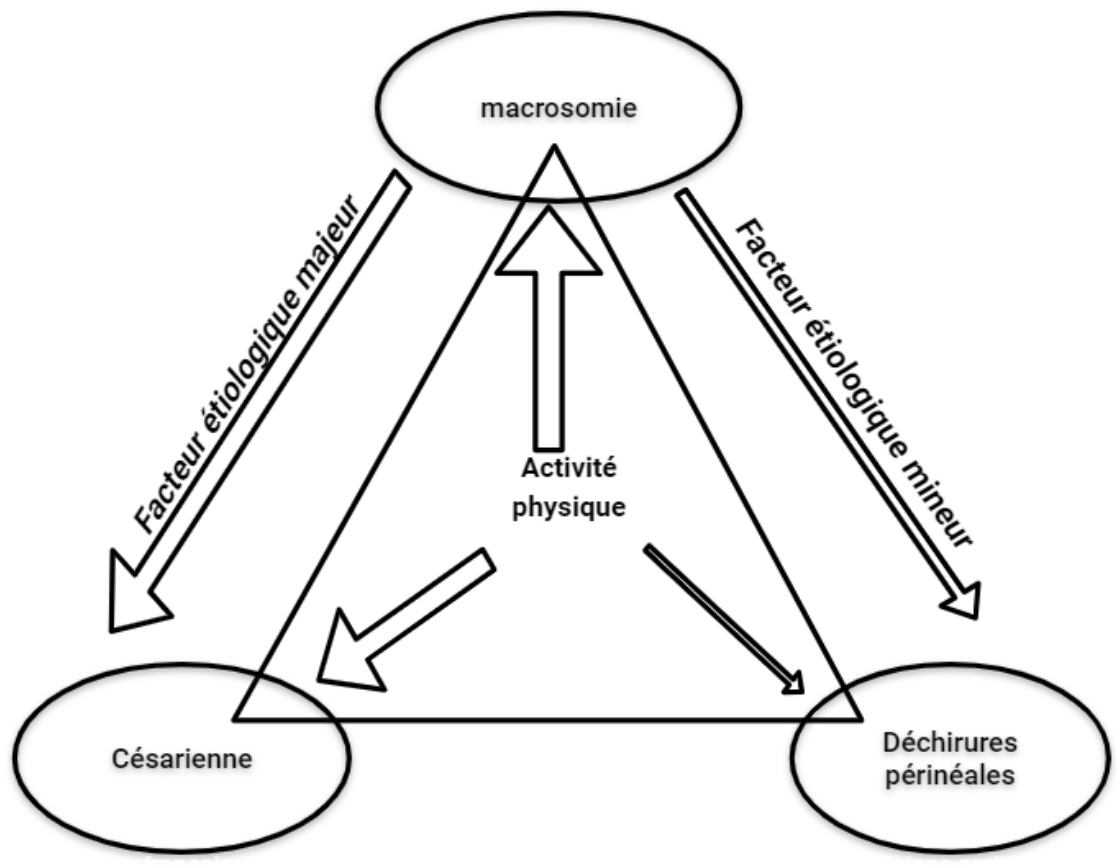

